\title{
Neodiplopeltula gen. nov. from the west coast of Sweden and reappraisal of the genus Diplopeltula Gerlach, 1950 (Nematoda, Diplopeltidae)
}

\author{
Oleksandr HOLOVACHOV ${ }^{1, *} \&$ Sven BOSTRÖM ${ }^{2}$ \\ ${ }^{1,2}$ Department of Zoology, Swedish Museum of Natural History, Box 50007, \\ SE-104 05 Stockholm, Sweden. \\ *Corresponding author: oleksandr.holovachov@nrm.se \\ 2Email: sven.bostrom@nrm.se \\ ${ }^{1}$ urn:1sid:zoobank.org:author:89D30ED8-CFD2-42EF-B962-30A13F97D203 \\ ${ }^{2}$ urn:Isid:zoobank.org:author:528300CC-D0F0-4097-9631-6C5F75922799
}

\begin{abstract}
The new genus Neodiplopeltula gen. nov. is proposed to accommodate those species from the genus Diplopeltula Gerlach, 1950 that possess the following morphological characters: amphids in the shape of an elongated loop, a well-developed subcylindrical stoma and outstretched ovaries. The genus Diplopeltula is considered genus inquirendum et incertae sedis. Four species placed in Neodiplopeltula gen. nov. are redescribed. The following taxonomic changes are proposed: Neodiplopeltula asymmetrica (Allgén, 1935) gen. et comb. nov.; Neodiplopeltula barentsi (Steiner, 1916) gen. et comb. nov.; Neodiplopeltula bathmanni (Jensen, 1991) gen. et comb. nov.; Neodiplopeltula cuspidiboja (Leduc, 2017) gen. et comb. nov.; Neodiplopeltula indica (Gerlach, 1962) gen. et comb. nov.; Neodiplopeltula intermedia (Gerlach, 1954) gen. et comb. nov.; Neodiplopeltula obesa (Nguyen Vu Thahn, Nguyen Thahn Hien \& Gagarin, 2012) gen. et comb. nov.; Neodiplopeltula onusta (Wieser, 1956) gen. et comb. nov.; Neodiplopeltula ovalis (Ditlevsen, 1928) gen. et comb. nov. and Neodiplopeltula tchesunovi (Fadeeva \& Mordukhovich, 2013) gen. et comb. nov. New synonyms include: Diplopeltis asymmetricus Allgén, 1935 and Diplopeltis ovalis Ditlevsen, 1928 are synonimised with Neodiplopeltula barentsi (Steiner, 1916) gen. et comb. nov.; Diplopeltula tchesunovi Fadeeva \& Mordukhovich, 2013 is synonimised with Neodiplopeltula onusta (Wieser, 1956) gen. et comb. nov.; the male of Diplopeltula cuspidiboja Leduc, 2017 is synonimised with Neodiplopeltula barentsi gen. et comb. nov. and the female with $N$. bathmanni gen. et comb. nov. A key to the species of Neodiplopeltula gen. nov. is provided.
\end{abstract}

Keywords. Identification key, morphology, revision, SEM, taxonomy.

Holovachov O. \& Boström S. 2018. Neodiplopeltula gen. nov. from the west coast of Sweden and reappraisal of the genus Diplopeltula Gerlach, 1950 (Nematoda, Diplopeltidae). European Journal of Taxonomy 458: 1-34. https://doi.org/10.5852/ejt.2018.458 


\section{Introduction}

The genus Diplopeltula Gerlach, 1950 was proposed for two new and one known species, but without an explicit generic diagnosis. Diagnostic characters of this genus mentioned in the identification key from the original publication (amphid is loop shaped, with equally long ventral and dorsal branches; amphid not located on the shield-like cuticular plate) match a number of genera currently included in the family Diplopeltidae, such as Morlaxia Vincx \& Gourbault, 1988; Mudwigglus Leduc, 2013; Pararaeolaimus Timm, 1961; Pseudaraeolaimus Chitwood, 1951 and even Araeolaimus de Man, 1888. Taxonomic instability of the genus is further sustained by the fact that the original description of the type species Diplopeltula breviceps Gerlach, 1950 does not include clear descriptions of characters currently considered diagnostic at the genus and family level, such as the exact structure of the pharynx or of the female reproductive system (Table 1), and because the type material was not preserved by S.A. Gerlach. As a result, the genus Diplopeltula recently underwent several taxonomic revisions, with more and more species being moved to other known and newly proposed genera (Holovachov et al. 2009; Leduc 2013; Holovachov 2017; Holovachov \& Boström 2017). In this manuscript we propose to move all the remaining properly described species from the genus Diplopeltula to a new genus Neodiplopeltula gen. nov., treat both Diplopeltula breviceps and D. longiceps Gerlach, 1950 as species inquirenda et incertae sedis, treat Diplopeltula ostrita Boucher \& Helléouët, 1977 and D. striolata Vincx \& Gourbault 1992 as species incertae sedis, and consider Diplopeltula a genus inquirendum et incertae sedis.

\section{Material and methods}

Bottom sediment samples were collected in several locations in the southern part of the Skagerrak and in the Gullmarn Fjord off the west coast of Sweden. All samples were collected with a bottom dredge or box corer and further sieved in the laboratory before fixation. Nematodes were extracted from samples using a decanting and sieving method (smallest mesh sizes: $45 \mu \mathrm{m}$ or $70 \mu \mathrm{m}$ ). Freshwater was used during sieving to induce an osmotic shock in nematodes inducing their detachment from the substrate. Material retained on the sieves was immediately fixed in a $4 \%$ formaldehyde solution in freshwater.

For light microscopy, formaldehyde-preserved specimens were transferred to pure glycerine using Seinhorst's (1959) rapid method as modified by De Grisse (1969). Permanent nematode mounts on glass slides were prepared using the paraffin wax ring method. After observations, six females and five males of $N$. incisa (Southern, 1914) gen. et comb. nov. from the sample \#S053 (SWEDEN: Skagerrak, $58^{\circ} 17^{\prime} 32^{\prime \prime} \mathrm{N}, 11^{\circ} 11^{\prime} 24^{\prime \prime}$ E, coarse sediment with algae at a deep of 45-55 m, 9 Aug. 2011, O. Holovachov leg.) were removed from slides and rehydrated by first gradually adding drops of S2 (5\% glycerine, 95\% ethanol) to glycerine in an embryo-dish, starting with a 1:4 ratio of S2 to glycerine, until the volume tripled, then gradually adding distilled water until the volume tripled again. The specimens were then washed in distilled water before resuspension in formaldehyde. For SEM, specimens were post-fixed in $1 \%$ osmium tetroxide $\left(\mathrm{OsO}_{4}\right)$ and transferred to pure acetone through an acetone/distilled water series. Specimens were critical point dried in liquid $\mathrm{CO}_{2}$, mounted on stubs, gold-plated under vacuum to a thickness of $200 \AA$ in an Agar High Resolution Sputter Coater Model 20, and examined in a Hitachi S-4300 SEM at an accelerating voltage of $10 \mathrm{kV}$.

All curved structures were measured along the curved median line. Measurements in all tables are presented in $\mu \mathrm{m}$ as mean and (range) where appropriate. Terminology follows Maggenti (2005). Specimens are deposited in the invertebrate collection of the Department of Zoology, Swedish Museum of Natural History, Stockholm, Sweden (SMNH). Type specimens of Diplopeltis asymmetricus Allgén, 1935 deposited in the Swedish Museum of Natural History in Stockholm, Diplopeltis ovalis Ditlevsen, 1928 deposited in the Natural History Museum of Denmark in Copenhagen and Diplopeltula ostrita Boucher \& Helléouët, 1977 deposited in the National Museum of Natural History in Paris were also examined. 
Table 1. Comparison of diagnostic characters between the genera Neodiplopeltula gen. nov., based on the type species $N$. incisa (Southern, 1914) gen. et comb. nov. and generic diagnosis (this publication), Diplopeltula Gerlach, 1950 based on the type species D. breviceps Gerlach, 1950, and Diplopeltoides Gerlach, 1962 based on the type species D. ornatus (Gerlach, 1950) and emended generic diagnosis in Holovachov \& Boström (2017).

\begin{tabular}{lccc}
\hline Family & Diplopeltidae & incertae sedis & Diplopeltoididae \\
Genus & Neodiplopeltula gen. nov. & Diplopeltula & Diplopeltoides \\
Stoma & subcylindrical & absent & small funnel-shaped \\
Pharynx & $\begin{array}{c}\text { uniformly cylindrical, } \\
\text { gradually widening } \\
\text { posteriorly, with evenly } \\
\text { distributed myofilaments }\end{array}$ & not described & $\begin{array}{c}\text { subdivided in anterior muscular corpus } \\
\text { with evenly distributed myofilaments and } \\
\text { posterior glandular postcorpus, consisting } \\
\text { of narrow isthmus and basal swelling }\end{array}$ \\
$\begin{array}{l}\text { Female } \\
\text { gonads }\end{array}$ & ovaries outstretched & not described & ovaries antidromously reflexed \\
\hline
\end{tabular}

\title{
Results
}

\author{
Phylum Nematoda Potts, 1932 \\ Class Chromadorea Inglis, 1983 \\ Order Araeolaimida De Coninck \& Schuurmans Stekhoven, 1933 \\ Family Diplopeltidae Filipjev, 1918 \\ Genus Neodiplopeltula gen. nov. \\ urn:1sid:zoobank.org:act:B1D9F161-1903-4227-8F97-5A2D6715F2CA
}

\section{Type species}

Neodiplopeltula incisa (Southern, 1914) gen. et comb. nov.

= Diplopeltis incisus Southern, 1914

= Diplopeltula incisa (Southern, 1914)

= Diplopeltis incisus sensu Gerlach, 1950 partim (note \#1)

= Diplopeltula incisa sensu Voronov, 1982 partim (note \#2)

\section{Diagnosis}

Cuticle transversely striated; striae visibly smooth under light microscope, may have fine longitudinal incisures visible under SEM. Lateral alae absent. Body pores and epidermal glands absent. Somatic sensilla present, most prominent along anterior part of pharyngeal region and on tail. Labial region bluntly rounded; lips fused. Six inner labial sensilla small, pore-like, located on anterior surface of lips, discernible under SEM only. Six outer labial sensilla papilliform, located on anterior surface of lips. Four cephalic sensilla setiform; their bases located at base of labial region, at level with anteriormost part of amphid. Bases of dorsosublateral setae often located more posteriorly than bases of ventrosublateral setae. Subcephalic sensilla absent. Cervical sensilla present in some species, setiform or papilliform, arranged in dorsosublateral and ventrosublateral rows at level with amphid and subcuticular periamphideal plates. Deirid and ocelli absent. Amphids in some species on strongly refractive lateral subcuticular periamphideal plates that can be connected together on ventral and dorsal sides forming a 'cephalic framework'. Amphidial fovea loop-shaped (inverted U-shaped), dorsal branch often longer than ventral branch; both branches of amphideal fovea closely adjacent. Secretory- 
excretory system present; renette cell located opposite to posterior part of pharynx or anterior part of intestine. Secretory-excretory ampulla present, located at level of pharynx. Cuticularised secretoryexcretory duct very short, opens to exterior either anterior or posterior to nerve ring level. Oral opening apical or shifted towards dorsal side of body. Buccal cavity usually well developed, subcylindrical and often asymmetrical: cheilostom usually broad, flexible; gymnostom subcylindrical, with weakly cuticularised walls; stegostom short conoid, its lining is uniform with lining of pharynx. Pharyngeal tubes absent. Pharynx subcylindrical, muscular, with evenly distributed myofilaments, gradually expanding towards posterior end; not subdivided into distinct sections; pharyngeal lumen uniform in thickness along entire pharynx length; valves absent. Cardia glandular, variable in shape and size, its posterior part embedded in intestinal tissue. Female reproductive system didelphic-amphidelphic, with equally developed branches, ovaries outstretched. Spermatheca present. Vulva equatorial or postquatorial. Vagina straight; pars proximalis vaginae encircled by large sphincter muscle; pars refringens vaginae absent, epiptygmata present or absent. Male reproductive system diorchic, testes opposed (anterior testis outstretched and posterior testis reflexed). Spicules symmetrical, arcuate; gubernaculum present, usually with strongly developed apophyses. Supplements, precloacal and postcloacal sensilla absent. Three caudal glands present, open via three separate openings, their cells and nuclei incaudal. Spinneret absent.

\section{Etymology}

The genus name is composed of a prefix 'neo-', meaning 'new' and Diplopeltula, and defines a new taxon proposed to accommodate species formerly placed in the genus Diplopeltula.

\section{Relationships}

The new genus differs from all other taxa currently included in the family Diplopeltidae (Holovachov 2017) in having amphids in the shape of an elongated loop, a well-developed subcylindrical stoma and outstretched ovaries. It is proposed in order to accommodate a number of species previously placed in the genus Diplopeltula and to solve a long lasting taxonomic conundrum (Vincx \& Gourbault 1992; Holovachov et al. 2009; Holovachov 2017; Holovachov \& Boström 2017). The description of Diplopeltula breviceps, type species of the genus Diplopeltula, does not include clear descriptions of several important taxonomic characters (see Table 1) and the absence of such data prevents an unequivocal placement of the species $D$. breviceps and the genus Diplopeltula in the classification system of Nematoda. As a result, $D$. breviceps is considered species inquirenda et incertae sedis and the genus Diplopeltula is accordingly considered genus inquirendum et incertae sedis. The second species, D. longiceps, is also considered species inquirenda et incertae sedis for the same reasons (incomplete description and unclear systematic position).

\section{Valid species}

Neodiplopeltula barentsi (Steiner, 1916) gen. et comb. nov.

= Diplopeltis barentsi Steiner, 1916

= Diplopeltis ovalis Ditlevsen, 1928 syn. nov. (note \#3)

= Neodiplopeltula ovalis (Ditlevsen, 1928) gen. et comb. nov., syn. nov.

= Diplopeltis asymmetricus Allgén, 1935 syn. nov. (note \#4)

= Neodiplopeltula asymmetrica (Allgén, 1935) gen. et comb. nov., syn. nov.

= Diplopeltis incisus sensu Gerlach, 1950 partim (note \#1)

= Diplopeltula incisa sensu Voronov, 1982 partim (note \#2)

= Diplopeltis ovalis sensu Sergeeva, 1977 (note \#5)

= Diplopeltula cuspidiboja Leduc, 2017 partim syn. nov. (note \#6)

= Neodiplopeltula cuspidiboja (Leduc, 2017) gen. et comb. nov., syn. nov. 
Neodiplopeltula bathmanni (Jensen, 1991) gen. et comb. nov.

= Diplopeltis bathmanni Jensen, 1991

= Diplopeltula bathmanni (Jensen, 1991)

= Diplopeltula incisa sensu Vitiello, 1972 (note \#7)

= Diplopeltula incisa sensu Voronov, 1982 partim (note \#2)

= Diplopeltula cuspidiboja Leduc, 2017 partim syn. nov. (note \#8)

= Neodiplopeltula cuspidiboja (Leduc, 2017) gen. et comb. nov., syn. nov.

Neodiplopeltula indica (Gerlach, 1962) gen. et comb. nov. (note \#9)

= Diplopeltula indica Gerlach, 1962

Neodiplopeltula intermedia (Gerlach, 1954) gen. et comb. nov. (note \#9)

= Diplopeltula intermedia Gerlach, 1954

Neodiplopeltula obesa (Nguyen Vu Thahn, Nguyen Thahn Hien \& Gagarin, 2012) gen. et comb. nov. = Diplopeltula obesa Nguyen Vu Thahn, Nguyen Thahn Hien \& Gagarin, 2012 (note \#9)

Neodiplopeltula onusta (Wieser, 1956) gen. et comb. nov.

= Diplopeltis onustus Wieser, 1956

= Diplopeltula onusta (Wieser, 1956)

= Diplopeltula incisa sensu Voronov, 1982 partim (note \#2)

= Diplopeltula tchesunovi Fadeeva \& Mordukhovich, 2013 syn. nov. (note \#10)

= Neodiplopeltula tchesunovi (Fadeeva \& Mordukhovich, 2013) gen. et comb. nov., syn. nov.

\section{Species inquirenda et incertae sedis}

Diplopeltula breviceps Gerlach, 1950

Diplopeltula longiceps Gerlach, 1950

\section{Species incertae sedis}

Diplopeltula striolata Vincx \& Gourbault, 1992 (note \#11)

Diplopeltula ostrita Boucher \& Helléouët, 1977 (note \#12)

\section{Nomenclatorial changes and notes}

1) The description of Diplopeltis incisus sensu Gerlach, 1950 is based on specimens collected in several localities in the Kiel Bay and is likely to be a mixture of at least two different species: based on the oval shape and relatively small size of the periamphideal refractive plate, the position of cephalic setae posterior to the oral opening, and the position of the oral opening close to the anterior end, $N$. barentsi gen. et comb. nov. can be identified on fig. 3h, 3k \& 3m in Gerlach (1950), while fig. 3b likely depicts $N$. incisa sensu stricto, based on the irregular shape and relatively large size of the periamphideal refractive plate, the position of cephalic setae posterior to the oral opening, and the position of the oral opening close to the anterior end. Unfortunately, exact measurements of morphological characters cannot be attained from the figures in Gerlach's publication due to the absence of proper scale bars. Thus, our hypothesis on the taxonomic identity of specimens studied by Gerlach (1950) must solely rely on illustrations and cannot be verified any further.

2) The description of Diplopeltula incisa sensu Voronov, 1982 is based on specimens collected in six different locations from the White Sea, within an area of more than $100 \times 200 \mathrm{~km}$ in size and ranging in depth from 50 to 250 meters. Many measurements given by Voronov vary within $2 \times-4 \times$ range. Morphological features of the anterior end also show a considerable variability (noted by Voronov himself) suggesting that his description is based on more than one morphospecies. Here 
we suggest that there are four different species of Neodiplopeltula gen. nov. illustrated in Voronov's publication: fig. 1a likely depicts $N$. bathmanni gen. et comb. nov. with very narrow periamphideal plates and an oral opening located at level with the cephalic setae bases; both specimens depicted on fig. 16 and 1в match our concept of $N$. barentsi gen. et comb. nov. with relatively broad oval periamphideal plates and an oral opening located close to the anterior terminus, anterior to the cephalic setae bases; the presence of irregularly shaped periamphideal plates and an oral opening located close to the anterior terminus, anterior to cephalic setae bases in specimen depicted on 1г suggest it to belong to $N$. incisa gen. et comb. nov.; while the anterior end shown on fig. 1д completely matches with $N$. onusta gen. et comb. nov. (and confirmed by Voronov in his figure caption) in having periamphideal plates merging on the dorsal and ventral body sides and an oral opening located at level with the cephalic setae bases.

3) The holotype of Diplopeltis ovalis described by Ditlevsen (1928) from Greenland is available in the collection of the Natural History Museum of Denmark in Copenhagen and is in rather acceptable shape. Some morphological features, such as cephalic setae and the spinneret cannot be seen due to deterioration, while the vagina and rectum are difficult to observe since the specimen is not in perfect lateral position on the slide. Morphological and morphometric features that are visible and measurable, show no differences from $N$. barentsi gen. et comb. nov. - in particular, the position of the oral opening and morphology of refractive plates underlying the amphids are identical (Figs 14A, 15A-B). Consequently, Diplopeltis ovalis is being transferred to the genus Neodiplopeltula gen. nov. and synonimised with $N$. barentsi gen. et comb. nov.

4) The lectotype of Diplopeltis asymmetricus described by Allgén (1935) from Öresund was found in the collection and is in poor shape. The specimen is partly dried, thus making it impossible to observe and measure smaller features such as cephalic setae, spinneret and rectum. It is also slightly flattened, which affects a- and c'-ratios. Morphological features that are visible, show no differences from $N$. barentsi gen. et comb. nov. - in particular, the position of the oral opening and the morphology of refractive plates underlying the amphids are identical (Figs 14B, 15C). Consequently, Diplopeltis asymmetricus is being transferred to the genus Neodiplopeltula gen. nov. and synonimised with $N$. barentsi gen. et comb. nov.

5) Diplopeltis ovalis sensu Sergeeva, 1977 is similar to $N$. barentsi gen. et comb. nov. in the position of the oral opening on the dorsal side of the body but close to the anterior end, length and arrangement of the cephalic setae (7.5 $\mu \mathrm{m}$ vs 3.5-7.5 $\mu \mathrm{m}$ in present specimens), and the shape of refractive plates (elongated ovoid, not connected).

6) The male of Diplopeltula cuspidiboja gen. et comb. nov. is identical to $N$. barentsi gen. et comb. nov. in the position of the oral opening, size and shape of refractive plates and the great majority of quantitative characters (see Table 3), except for a slightly longer amphid (32 $\mu \mathrm{m}$ vs $21-25.5 \mu \mathrm{m}$ ) and gubernaculum $(20 \mu \mathrm{m}$ vs $10-11.5 \mu \mathrm{m})$.

7) Diplopeltula incisa sensu Vitiello, 1972 is similar to D. bathmanni in the position of the oral opening, length and the arrangement of the cephalic setae, the size and position of the periamphideal refractive plate, length of the tail and position of the caudal gland openings.

8) The female of Diplopeltula cuspidiboja gen. et comb. nov. is identical to N. bathmanni gen. et comb. nov. in the position of the oral opening, the position of the cephalic sensilla, the size and shape of the refractive plates (Leduc 2017); as a result of having a larger body (2076 $\mu \mathrm{m}$ vs $1270-1673 \mu \mathrm{m})$, many morphometric characters of the female of $D$. cuspidiboja are also outside 
the measurement range of both Nordic populations, which can be attributed to geographic variability.

9) Diplopeltula indica and Diplopeltula intermedia both match the diagnostic characters of the genus Neodiplopeltula gen. nov.: amphids in the shape of an elongated loop, a well-developed subcylindrical stoma and outstretched ovaries. Diplopeltula obesa also has amphids in the shape of an elongated loop and outstretched ovaries, however, the structure of the stoma in this species is described as "not expressed", leaving some doubts as to its exact morphology.

10) Diplopeltula tchesunovi is identical to D. onusta in all qualitative and quantitative characters (Table 4), with the exception of longer refractive plates (41-48 $\mu \mathrm{m}$ vs 32-38 $\mu \mathrm{m}$ ), which can be attributed to geographic variability.

11) Diplopeltula striolata is described based only on one male, thus the structure of the female reproductive system remains unknown, questioning its systematic affinities. It is similar to the genus Neodiplopeltula gen. nov. in the morphology of the amphid (in the shape of an elongated loop) and stoma (small but well developed, subcylindrical). The morphology of the pharynx (uniformly cylindrical) also matches that of Neodiplopeltula gen. nov. However, D. striolata strongly differs from Neodiplopeltula gen. nov. in having a strongly annulated cuticle, the position of the excretory pore at the level with the anterior part of the intestine, the presence of a spinneret, the absence of a gubernaculum. Although this species can easily be identified, and its validity is unquestionable, its taxonomic position remains unresolved as it cannot be assigned to any of the existing genera with confidence (species incertae sedis).

12) All available type specimens of Diplopeltula ostrita were examined, but not all morphological characters can be observed. That includes the female reproductive system and the structure of the ovaries. However, D. ostrita strongly differs from Neodiplopeltula gen. nov. in having a strongly annulated cuticle, a minute buccal cavity, weak spicules and gubernaculum. Similar to Diplopletula striolata, D. ostrita is here considered to be a valid species, which, however, cannot be assigned to any existing genus with confidence and must be considered species incertae sedis.

Neodiplopeltula incisa (Southern, 1914) gen et. comb. nov.

Figs 1-4; Table 2

Diagnosis (based on combined data)

Neodiplopeltula incisa gen. et comb. nov. is characterised by a $1.52-2.24 \mathrm{~mm}$ long body; refractive plates underlying cephalic cuticle around amphids present, irregular in shape, not joined, 28-33 $\mu \mathrm{m}$ long and 21-25 $\mu \mathrm{m}$ wide; cephalic setae 8-14 $\mu \mathrm{m}$ long; amphidial fovea 22-28 $\mu \mathrm{m}$ long and 5.5-7 $\mu \mathrm{m}$ wide; oral opening 1-3 $\mu \mathrm{m}$ from anterior end, posterior to cephalic setae bases; secretory-excretory pore located opposite to posterior part of pharynx; tail 2.5-3.9 anal body diameters long; vagina with epiptygmata; spicules $66-79 \mu \mathrm{m}$ long.

\section{Material examined}

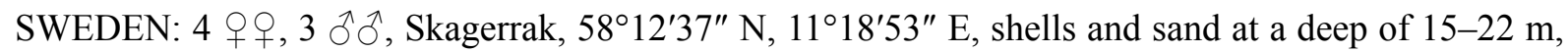

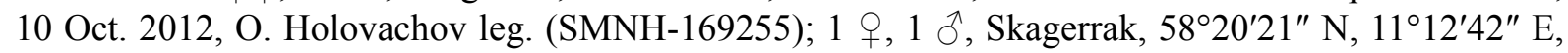
coarse shell sand at a deep of 14-17 m, 19 Aug. 2014, O. Holovachov leg. (SMNH-169258). 

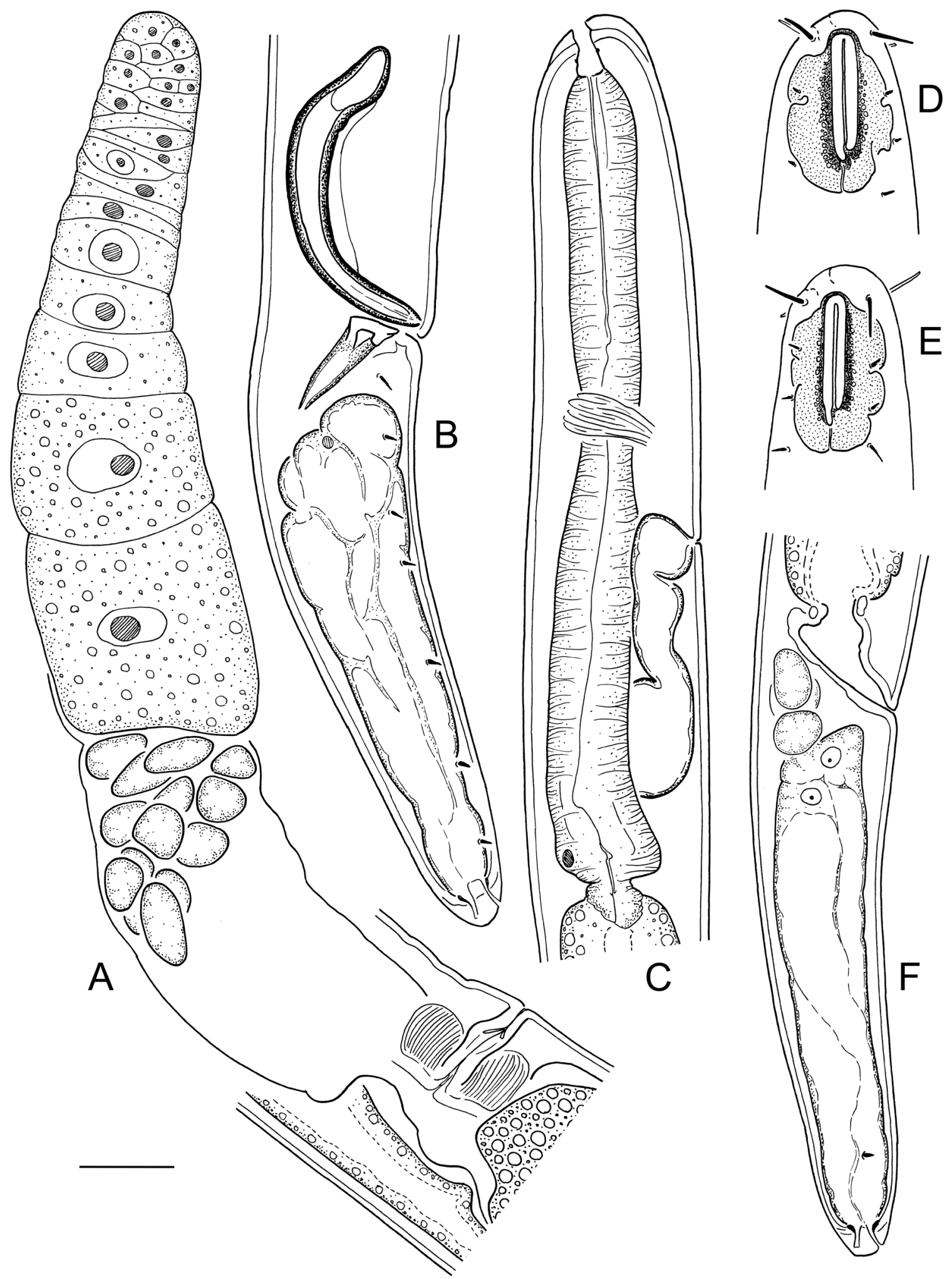

Fig. 1. Neodiplopeltula incisa (Southern, 1914) gen. et comb. nov. (SMNH-169255). A. Anterior part of female reproductive system. B. Male tail. C. Female pharyngeal region, optical median section. D. Female anterior end, surface view. E. Male anterior end, surface view. F. Female tail. Scale bar = $20 \mu \mathrm{m}$. 


\section{Description}

\section{Adult}

Body cylindrical, posteriorly tapering in tail region, straight or weakly ventrally curved upon fixation. Cuticle finely transversely striated along entire body as seen under SEM (striation can be observed under LM but very fine and shallow and cannot be measured with confidence), except for visually smooth labial region and terminal part of tail; longitudinal striation not observed under the light

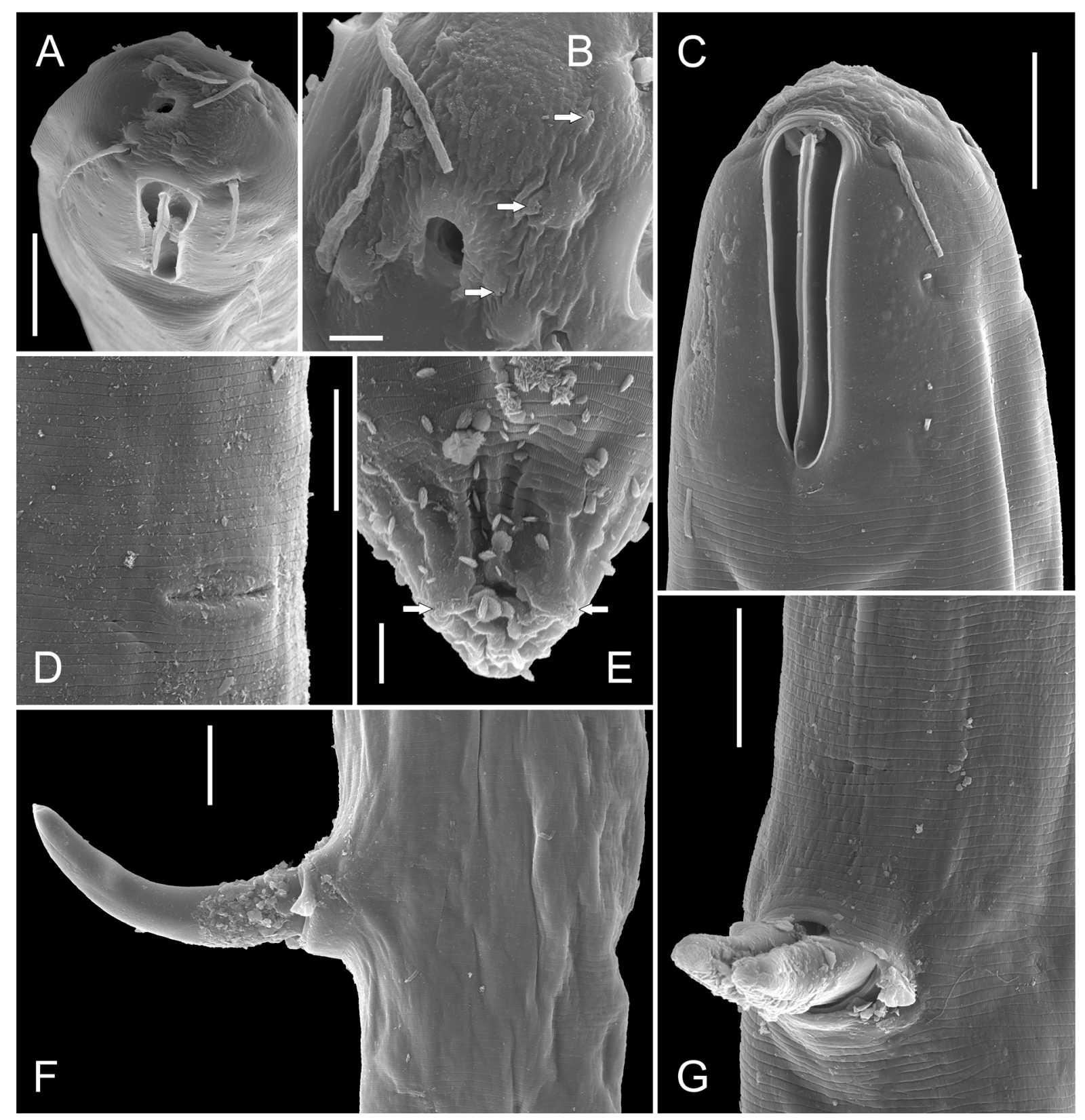

Fig. 2. Neodiplopeltula incisa (Southern, 1914) gen. et comb. nov., SEM micrographs. A-C, F-G. O̊. D-E. . . A. Male anterior end, semi-apical view, dorsal side to the left. B. Male labial region, semiapical view, dorsal side down (arrows point at inner labial sensilla). C. Male anterior end, right lateral view. D. Vulval region. E. Tail terminus. F. Male cloacal region, lateral view. G. Male cloacal region, ventrosubventral view. Scale bars: A, C-D, F-G $=10 \mu \mathrm{m} ; \mathrm{B}, \mathrm{E}=2 \mu \mathrm{m}$. 
Table 2 (continued on next page). Measurements (in $\mu \mathrm{m}$ ) of Neodiplopeltula incisa (Southern, 1914) gen. et comb. nov.

\begin{tabular}{|c|c|c|c|c|}
\hline & \multicolumn{2}{|c|}{ original description } & \multicolumn{2}{|c|}{ recent specimens } \\
\hline & q & $2 \widehat{\partial}$ & 5 우우 & 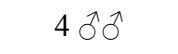 \\
\hline Body length & 2020 & $2100-2240$ & $\begin{array}{c}1740 \pm 176 \\
(1515-2001)\end{array}$ & $\begin{array}{c}1748 \pm 131 \\
(1591-1908)\end{array}$ \\
\hline Body diameter (BD) & 62 & $53-58$ & $\begin{array}{c}48 \pm 7 \\
(42-59)\end{array}$ & $\begin{array}{c}45 \pm 9 \\
(34-53)\end{array}$ \\
\hline Pharynx length & 270 & $250-270$ & $\begin{array}{c}204 \pm 24 \\
(186-245)\end{array}$ & $\begin{array}{c}216 \pm 31 \\
(178-254)\end{array}$ \\
\hline Tail length & 124 & $124-136$ & $\begin{array}{c}122 \pm 10 \\
(106-135)\end{array}$ & $\begin{array}{c}133 \pm 13 \\
(116-147)\end{array}$ \\
\hline Anal body diameter (ABD) & 45 & 50 & $\begin{array}{c}35 \pm 3 \\
(32-39)\end{array}$ & $\begin{array}{c}37 \pm 1 \\
(37-39.0)\end{array}$ \\
\hline $\mathrm{a}$ & 36.7 & $38.6-39.6$ & $\begin{array}{c}36.9 \pm 4.3 \\
(30.0-40.8)\end{array}$ & $\begin{array}{c}39.9 \pm 6.1 \\
(32.8-46.5)\end{array}$ \\
\hline $\mathrm{b}$ & 7.5 & $8.3-8.4$ & $\begin{array}{l}8.6 \pm 0.7 \\
(7.7-9.6)\end{array}$ & $\begin{array}{l}8.2 \pm 0.6 \\
(7.5-8.9)\end{array}$ \\
\hline $\mathrm{c}$ & 16.3 & $16.5-17$ & $\begin{array}{c}14.4 \pm 1.6 \\
(12.4-16.8)\end{array}$ & $\begin{array}{c}13.1 \pm 0.4 \\
(12.9-13.7)\end{array}$ \\
\hline c' & 2.8 & 2.5 & $\begin{array}{l}3.5 \pm 0.3 \\
(2.9-3.8)\end{array}$ & $\begin{array}{l}3.6 \pm 0.3 \\
(3.2-3.9)\end{array}$ \\
\hline $\mathrm{V}(\%) / \mathrm{T}(\%)$ & 61 & $?$ & $\begin{array}{c}62.7 \pm 0.4 \\
(62.1-62.9)\end{array}$ & $\begin{array}{c}45.8 \pm 5.1 \\
(40.4-51.6)\end{array}$ \\
\hline Labial region diameter & $?$ & $?$ & $\begin{array}{c}19.6 \pm 1.6 \\
(18.0-22.0)\end{array}$ & $\begin{array}{c}20.9 \pm 1.1 \\
(20.0-22.0)\end{array}$ \\
\hline Cephalic setae length & $?$ & $?$ & $\begin{array}{l}10.6 \pm 2.2 \\
(8.0-14.0)\end{array}$ & $\begin{array}{c}11.0 \pm 1.0 \\
(10.5-12.0)\end{array}$ \\
\hline Subdorsal cephalic setae from anterior end & $?$ & $?$ & $\begin{array}{c}7.2 \pm 2.0 \\
(5.5-10.5)\end{array}$ & $\begin{array}{l}6.4 \pm 0.8 \\
(5.5-7.0)\end{array}$ \\
\hline Subventral cephalic setae from anterior end & $?$ & $?$ & $\begin{array}{c}7.2 \pm 2.0 \\
(5.5-10.5)\end{array}$ & $\begin{array}{l}6.4 \pm 0.8 \\
(5.5-7.0)\end{array}$ \\
\hline Cephalic setae length / labial region diam. & $?$ & $?$ & $\begin{array}{l}0.5 \pm 0.1 \\
(0.4-0.8)\end{array}$ & 0.5 \\
\hline Anterior end of plate from anterior end & $?$ & $?$ & $\begin{array}{c}9.5 \pm 1.0 \\
(8.0-10.5)\end{array}$ & 10.0 \\
\hline Refractive plate length & $?$ & $?$ & $\begin{array}{c}31.4 \pm 2.1 \\
(28.0-33.0)\end{array}$ & $\begin{array}{c}32.0 \pm 1.1 \\
(31.0-33.0)\end{array}$ \\
\hline Refractive plate width & $?$ & $?$ & $\begin{array}{c}23.6 \pm 2.0 \\
(21.0-25.0)\end{array}$ & $\begin{array}{c}23.2 \pm 0.8 \\
(23.0-24.0)\end{array}$ \\
\hline Anterior end of amphid from anterior end & $?$ & $?$ & $\begin{array}{l}6.2 \pm 1.2 \\
(4.0-7.0)\end{array}$ & $\begin{array}{l}6.4 \pm 0.8 \\
(5.5-7.0)\end{array}$ \\
\hline Dorsal amphid branch length & $?$ & $?$ & $\begin{array}{c}27.3 \pm 1.8 \\
(25.5-30.0)\end{array}$ & $\begin{array}{c}28.5 \pm 1.6 \\
(27.6-30.4)\end{array}$ \\
\hline Ventral amphid branch length & $?$ & $?$ & $\begin{array}{c}25.4 \pm 2.3 \\
(22.0-27.0)\end{array}$ & $\begin{array}{c}27.6 \pm 0.7 \\
(26.9-28.3)\end{array}$ \\
\hline Amphid width & $?$ & $?$ & $\begin{array}{l}5.7 \pm 0.3 \\
(5.5-6.0)\end{array}$ & $\begin{array}{l}6.2 \pm 0.7 \\
(5.5-6.9)\end{array}$ \\
\hline Amphid length / width & $?$ & $?$ & $\begin{array}{l}4.8 \pm 0.2 \\
(4.6-5.0)\end{array}$ & $\begin{array}{l}4.6 \pm 0.3 \\
(4.4-5.0)\end{array}$ \\
\hline
\end{tabular}


Table 2 (continued).

\begin{tabular}{|c|c|c|c|c|}
\hline \multirow[b]{2}{*}{ Stoma length } & \multicolumn{2}{|c|}{ original description } & \multicolumn{2}{|c|}{ recent specimens } \\
\hline & $?$ & $?$ & $\begin{array}{c}14.4 \pm 1.8 \\
(12.5-17.0)\end{array}$ & $\begin{array}{c}16.3 \pm 0.8 \\
(16.0-17.0)\end{array}$ \\
\hline Stoma width & $?$ & $?$ & $\begin{array}{l}5.7 \pm 0.3 \\
(5.5-6.0)\end{array}$ & $\begin{array}{l}6.0 \pm 0.4 \\
(5.5-6.0)\end{array}$ \\
\hline Nerve ring from anterior end & $?$ & $111-115$ & $\begin{array}{l}102 \pm 10 \\
(94-114)\end{array}$ & $\begin{array}{l}106 \pm 12 \\
(91-120)\end{array}$ \\
\hline Nerve ring from anterior end ( $\%$ pharynx) & $?$ & $42.6-44.4$ & $\begin{array}{c}49.7 \pm 3.1 \\
(46.5-53.8)\end{array}$ & $\begin{array}{r}49.4 \pm 1.7 \\
(47.1-51.3)\end{array}$ \\
\hline Secretory-excretory pore from ant. end & $?$ & $?$ & $\begin{array}{c}118 \pm 13 \\
(108-140)\end{array}$ & $\begin{array}{l}125 \pm 19 \\
(99-143)\end{array}$ \\
\hline Secr.-excr. pore from ant. end ( $\%$ pharynx) & $?$ & $?$ & $\begin{array}{c}58.2 \pm 1.4 \\
(57.0-60.6)\end{array}$ & $\begin{array}{c}57.8 \pm 2.2 \\
(55.8-60.4)\end{array}$ \\
\hline Vagina or spicules length & $?$ & 66 & $\begin{array}{c}24.8 \pm 3.3 \\
(23.0-29.5)\end{array}$ & $\begin{array}{r}77.5 \pm 1.6 \\
(75.0-79.0)\end{array}$ \\
\hline Vagina / BD or spicules / ABD & $?$ & 1.3 & 0.5 & $\begin{array}{l}2.1 \pm 0.1 \\
(1.9-2.2)\end{array}$ \\
\hline Rectum or gubernaculum length & $?$ & $?$ & $\begin{array}{c}29.6 \pm 3.2 \\
(27.5-35.0)\end{array}$ & $\begin{array}{c}12.3 \pm 0.6 \\
(11.5-12.5)\end{array}$ \\
\hline Rectum / ABD or apophysis length & $?$ & $?$ & $\begin{array}{l}0.8 \pm 0.1 \\
(0.8-0.9)\end{array}$ & $\begin{array}{r}27.4 \pm 1.3 \\
(26.0-28.5)\end{array}$ \\
\hline Caudal gland openings from tail terminus & $?$ & $?$ & $\begin{array}{l}2.9 \pm 0.7 \\
(2.0-3.5)\end{array}$ & 2.0 \\
\hline
\end{tabular}

microscope, but distinct under the scanning electron microscope, covers cuticle over entire body length. Somatic sensilla visible along pharyngeal region (cervical setae, see below) and on tail. Labial region bluntly rounded, lips fused. Refractive plates underlying cephalic cuticle around amphids present (periamphideal), extending from level of anteriormost edge of amphid posteriorly some distance behind posteriormost edge of amphid; refractive plates irregular in shape (with incised edges), not connected with each other on ventral and dorsal sides. Inner labial sensilla small pore-like, located on anterior surface of lips, discernible under SEM only. Outer labial sensilla papilliform, located on anterior surface of lips, clearly visible under SEM only. Cephalic sensilla setiform, bases of dorsosublateral setae located posterior to oral opening. Cervical sensilla papilliform, arranged in four sublateral rows starting at level with middle of amphid and ending at level with posterior edge of refractive plate, two or three per row. Amphids similar in shape and size between sexes: amphidial fovea inverted U-shape with dorsal branch 0.5-3.5 $\mu \mathrm{m}$ longer than ventral branch. Oral opening shifted towards dorsal side of body. Stoma barrel-shaped: cheilostom broad; gymnostom barrel-shaped, with weakly cuticularised walls; stegostom short conoid, its lining uniform with lining of pharynx. Pharyngeal tubes absent. Pharynx subcylindrical, muscular, with evenly distributed myofilaments, gradually expanding towards posterior end; not subdivided in distinct sections; pharyngeal lumen uniform in thickness along entire pharynx length; valves absent. Cardia ovoid, almost entirely embedded into intestinal tissue. Secretory-excretory system present; secretory-excretory pore located along ventral body line opposite to posterior $3 / 5^{\text {th }}$ of pharynx; secretory-excretory duct very short, leading from pore to ampulla; renette cell small, its body adjacent and ventral to posterior part of pharynx. Tail cylindro-conical with bluntly rounded terminus. Caudal glands opening via three separate subterminal openings, spinneret absent. 


\section{Female}

Reproductive system didelphic, amphidelphic, ovary branches outstretched and symmetrical, on opposite sides of intestine. Anterior genital tube 248-280 $\mu \mathrm{m}$ long, situated to either right $(\mathrm{n}=1)$ or left $(n=4)$ of intestine; posterior genital tube 224-259 $\mu \mathrm{m}$ long, situated to either left $(n=1)$ or right

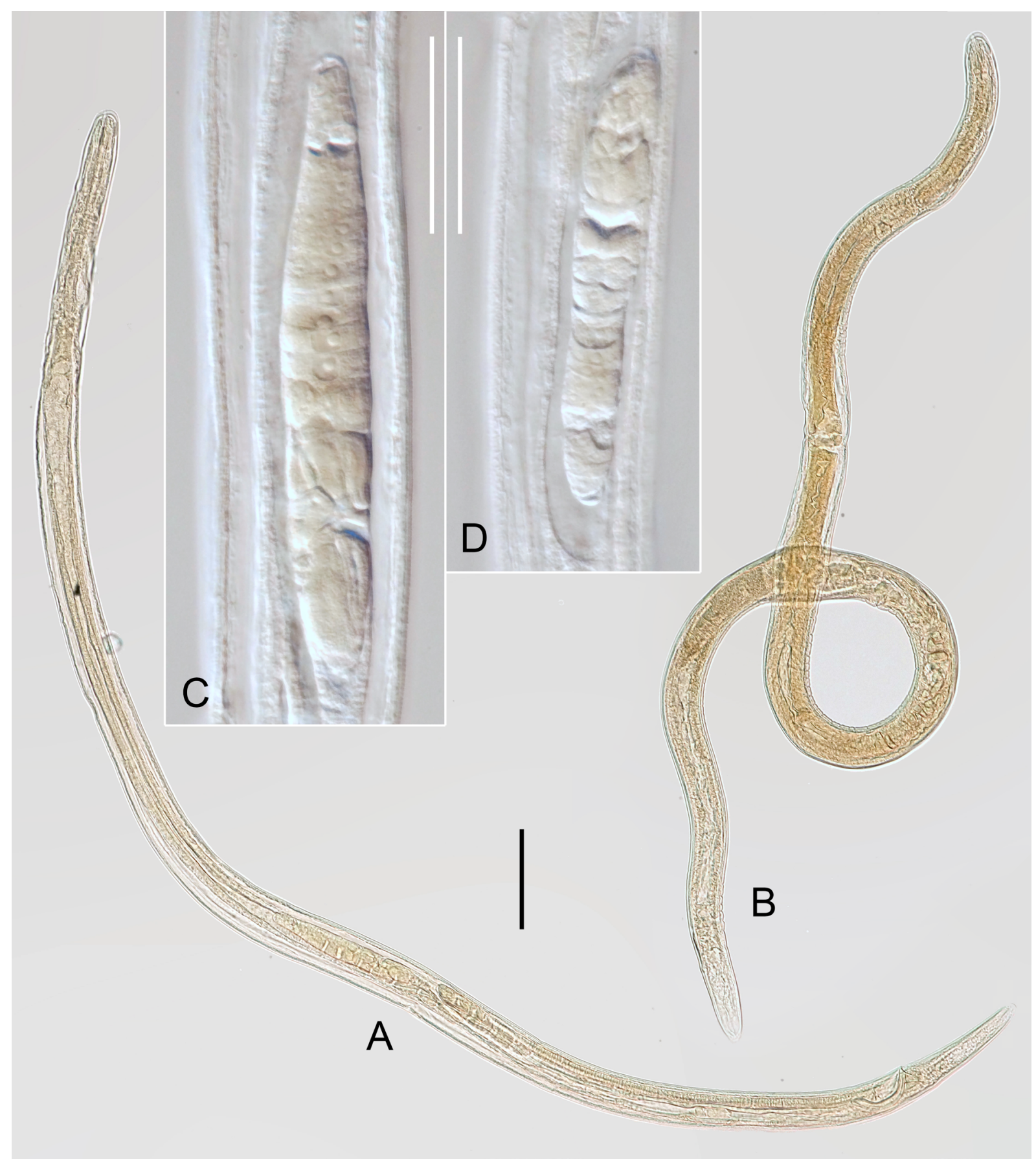

Fig. 3. Neodiplopeltula incisa (Southern, 1914) gen. et comb. nov. (SMNH-169255). A. $\widehat{\partial}$, entire specimen. B. , entire specimen. C. Anterior testis. D. Posterior testis. Scale bars: A-B $=100 \mu \mathrm{m}$; $\mathrm{C}-\mathrm{D}=50 \mu \mathrm{m}$. 
$(\mathrm{n}=4)$ of intestine. Vulva a transverse slit, located posterior to midbody. Vagina straight, with developed sphincter muscle surrounding proximal part and distinct epiptygmata in distal part; pars refringens vaginae absent. Sack-like spermatheca present, filled with oval spermatozoa in fertilized specimens. Rectum short.

\section{Male}

Reproductive system diorchic, testes opposed; anterior testis 162-286 $\mu \mathrm{m}$ long, outstretched and posterior testis 117-162 $\mu \mathrm{m}$ long, reflexed. Spicules paired and symmetrical, strongly curved, with ovoid manubrium and subcylindrical shaft. Gubernaculum plate-like, with pair of strong, closely set caudal apophyses variable in shape (Fig. 4C-E). Caudal setae present, several ventrosublateral and dorsosublateral pairs arranged in rows along entire tail length (often difficult to observe).

\section{Remarks}

Despite the fact that the original description by Southern (1914) is rather brief, the present population closely resembles the type specimens in most morphological and morphometric features, including the shape of the refractive plates underlying the amphids (described as "shield shaped" with "front and lateral walls are notched"). Spicules are slightly shorter in the type specimens (66 $\mu \mathrm{m}$ vs $75-79 \mu \mathrm{m}$ in recent specimens).

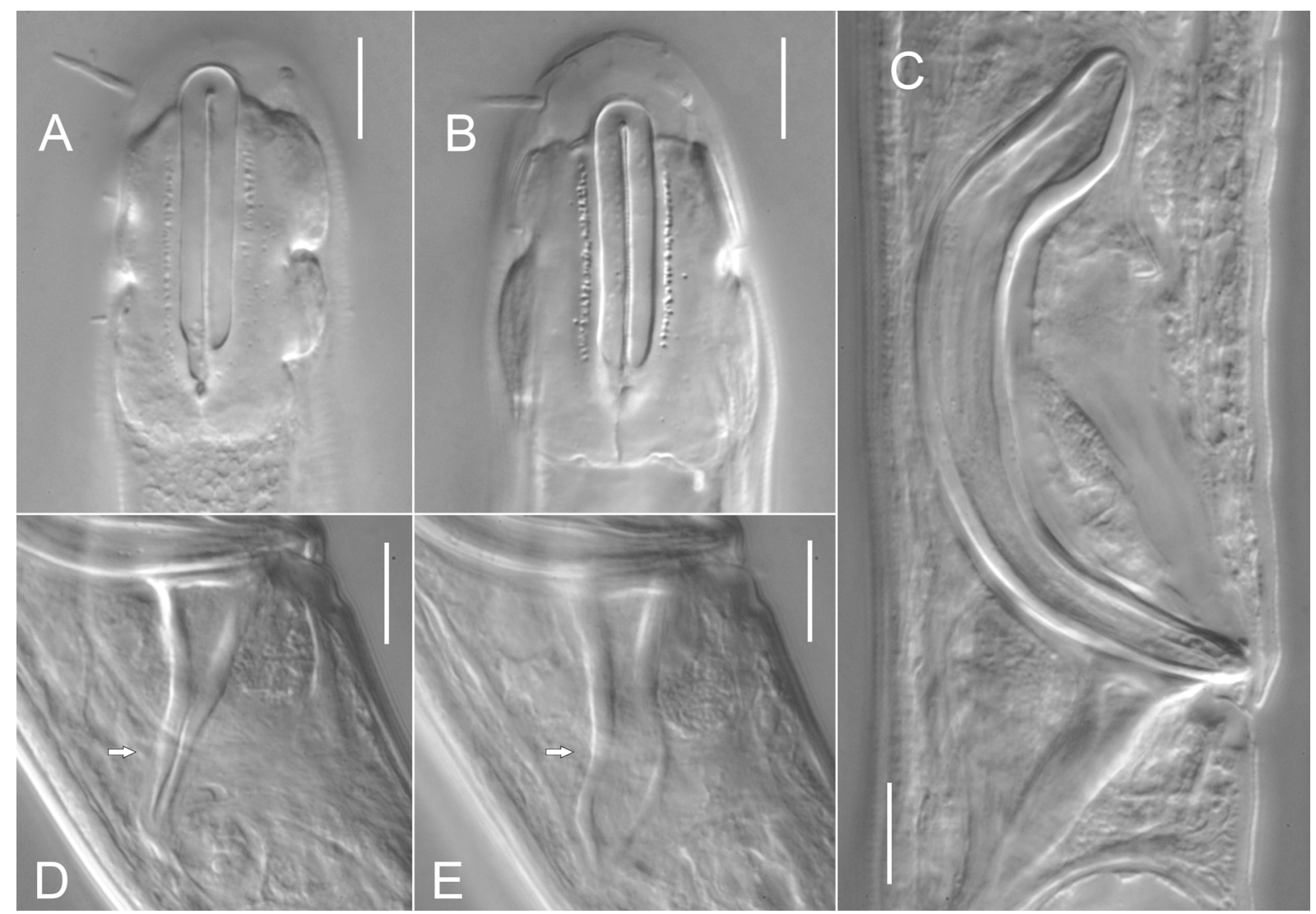

Fig. 4. Neodiplopeltula incisa (Southern, 1914) gen. et comb. nov. (SMNH-169255). A. Male anterior end, lateral view. B. Female anterior end, lateral view. C. Spicules and gubernaculum. D-E. Variability in the shape of gubernaculum apophyses in the same specimen (arrows). Scale bars $=10 \mu \mathrm{m}$. 
Neodiplopeltula barentsi (Steiner, 1916) gen. et comb. nov.

Figs 5-7, 14-15; Table 3

Diagnosis (based on combined data)

Neodiplopeltula barentsi gen. et comb. nov. is characterised by a $0.94-1.67 \mathrm{~mm}$ long body; refractive plates underlying cephalic cuticle around amphids present, elongated ovoid in shape, not joined, 23.5$32 \mu \mathrm{m}$ long and 12-16 $\mu \mathrm{m}$ wide; cephalic setae 3.5-7.5 $\mu \mathrm{m}$ long; amphidial fovea $21-32 \mu \mathrm{m}$ long and 4-7 $\mu \mathrm{m}$ wide; oral opening 1-2 from anterior end, posterior to cephalic setae bases; secretory-excretory

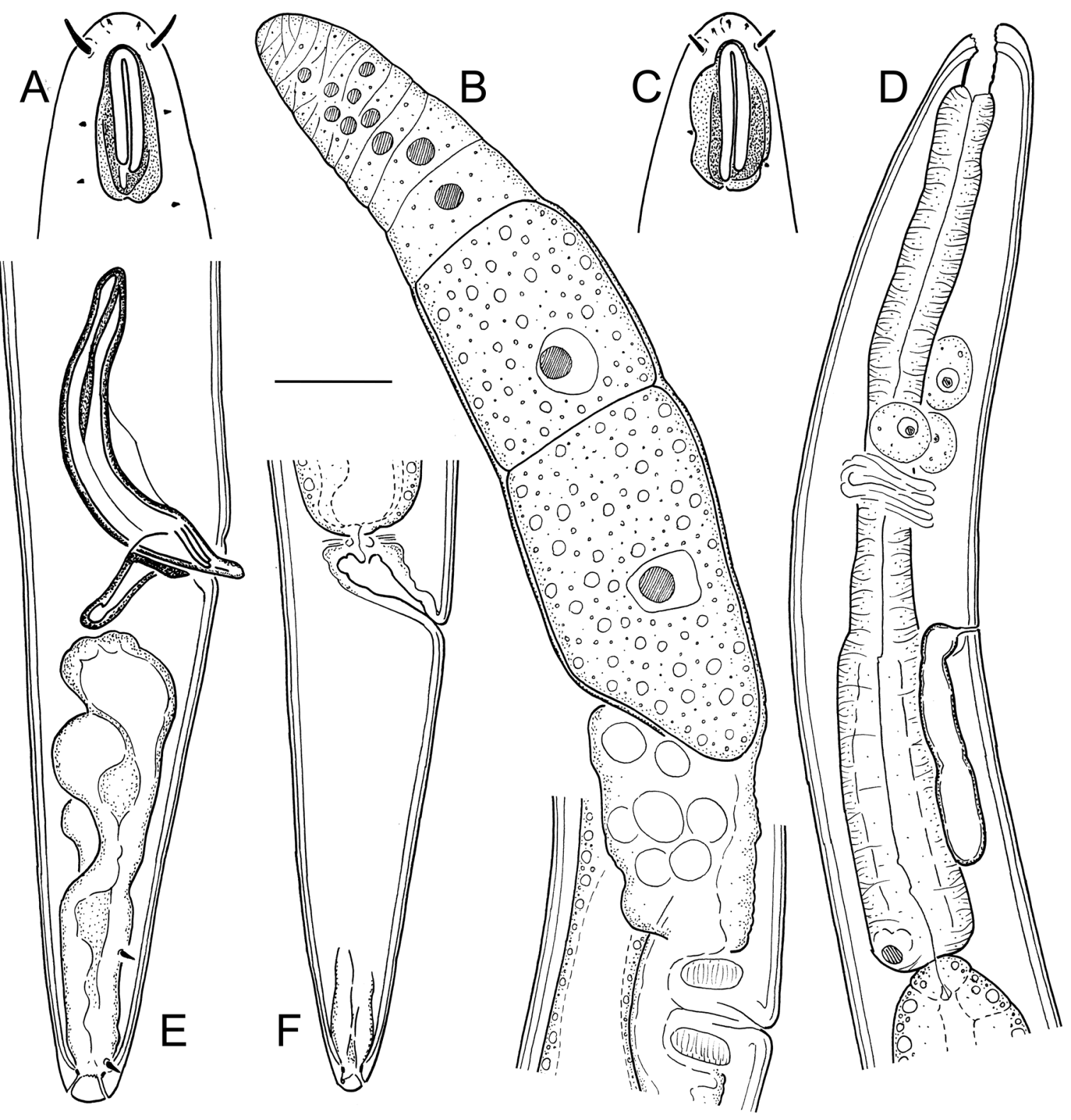

Fig. 5. Neodiplopeltula barentsi (Steiner, 1916) gen. et comb. nov. (SMNH-169268). A. Male anterior end, surface view. B. Anterior part of female reproductive system. C. Female anterior end, surface view. D. Female pharyngeal region, optical median section. E. Male tail. F. Female tail. Scale bar $=20 \mu \mathrm{m}$. 
pore located opposite to posterior part of pharynx; tail 1.6-3.3 anal body diameters long; vagina with epiptygmata; spicules $45-73 \mu \mathrm{m}$ long.

\section{Material examined}

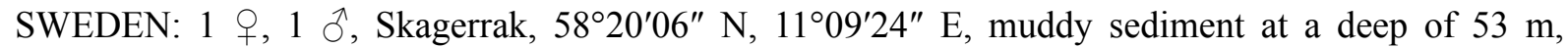

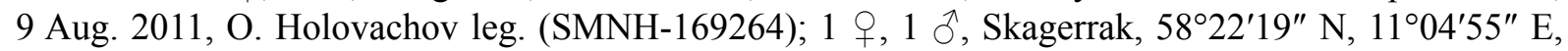
muddy sediment at a deep of 55-70 m, 9 Aug. 2011, O. Holovachov leg. (SMNH-169266); 3 q +1 ô,

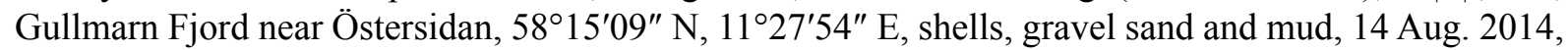
O. Holovachov leg. (SMNH-169268); 1 lectotype of Diplopeltis asymmetricus, Öresund, west of Valgrundet, at a deep of 30-36 m, Zostera and Echinocardium, 7 Aug. 1926 (SMNH Type-9015).

GREENLAND: 1 , holotype of Diplopeltis ovalis, Godhavn, mud, 17 Aug. 1926, Reisinger and Steinböck leg. (NHMD 102171).

\section{Description}

\section{Adult}

Body cylindrical, posteriorly tapering in tail region, straight or weakly ventrally curved upon fixation. Cuticle finely transversely striated along entire body, except for visually smooth labial region and terminal

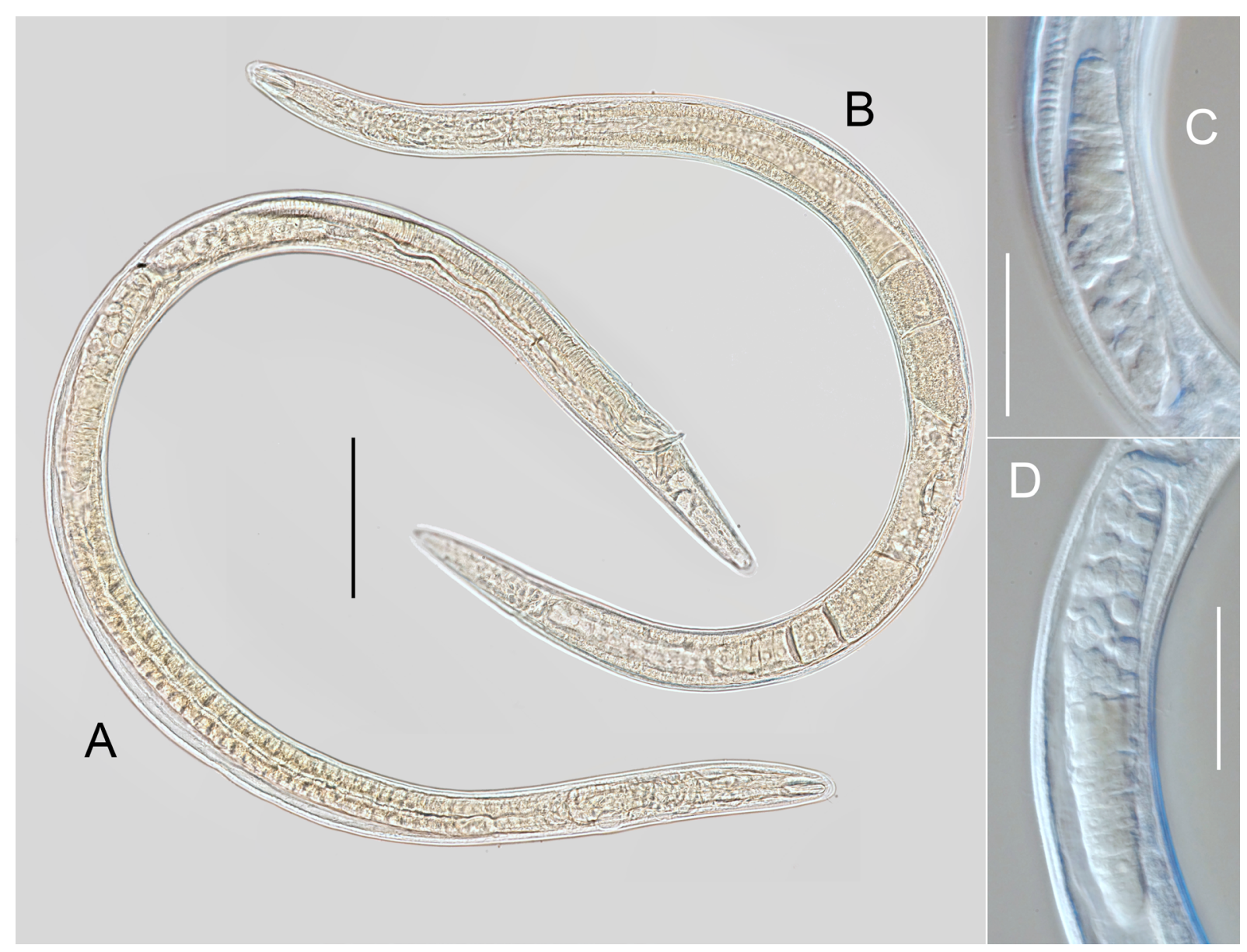

Fig. 6. Neodiplopeltula barentsi (Steiner, 1916) gen. et comb. nov. (SMNH-169268). A. $\hat{\partial}$, entire specimen. B. , entire specimen. C. Anterior testis. D. Posterior testis. Scale bars: A-B $=100 \mu \mathrm{m}$; $\mathrm{C}-\mathrm{D}=50 \mu \mathrm{m}$. 
Table 3 (continued on next page). Measurements (in $\mu \mathrm{m}$ ) of Neodiplopeltula barentsi (Steiner, 1916) gen. et comb. nov.

\begin{tabular}{|c|c|c|c|c|c|c|c|c|}
\hline & \multirow{2}{*}{$\begin{array}{c}\begin{array}{c}\text { original } \\
\text { description }\end{array} \\
0\end{array}$} & \multirow{2}{*}{$\begin{array}{c}\text { D. ovalis } \\
\text { q holotype }\end{array}$} & \multirow{2}{*}{$\begin{array}{c}\begin{array}{c}\text { D. } \\
\text { asymmetricus }\end{array} \\
+ \text { lectotype }\end{array}$} & \multicolumn{2}{|c|}{$\begin{array}{l}\text { D. onustus } \\
\text { sensu } \\
\text { Sergeeva } \\
(1974)\end{array}$} & \multirow{2}{*}{$\begin{array}{c}\text { data for } D . \\
\text { cuspidiboja } \\
\widehat{O}\end{array}$} & \multicolumn{2}{|c|}{ recent specimens } \\
\hline & & & & 우 & $\hat{0}$ & & 5 우우 & $3 \widehat{\partial}$ \\
\hline Body length & 1526 & 1228 & 970 & 1057 & 1147 & 1147 & $\begin{array}{l}1207 \pm 258 \\
(935-1532)\end{array}$ & $\begin{array}{c}1487 \pm 285 \\
(1159-1670)\end{array}$ \\
\hline Body diameter (BD) & $?$ & 61 & 65 & 39 & 45 & 44 & $\begin{array}{l}52 \pm 13 \\
(41-74)\end{array}$ & $\begin{array}{l}55 \pm 15 \\
(38-65)\end{array}$ \\
\hline Pharynx length & 205 & 197 & 159 & 169 & 180 & 175 & $\begin{array}{c}176 \pm 11 \\
(168-193)\end{array}$ & $\begin{array}{l}182 \pm 15 \\
(165-195)\end{array}$ \\
\hline Tail length & 122 & 95 & 64 & 73 & 79 & 67 & $\begin{array}{c}84 \pm 8 \\
(76-96)\end{array}$ & $\begin{array}{c}98 \pm 9 \\
(91-108)\end{array}$ \\
\hline $\begin{array}{l}\text { Anal body diameter } \\
\text { (ABD) }\end{array}$ & $?$ & 38 & 40 & 22 & 34 & 32 & $\begin{array}{c}35 \pm 6 \\
(29-43)\end{array}$ & $\begin{array}{c}41 \pm 6 \\
(34-46)\end{array}$ \\
\hline $\mathrm{a}$ & $?$ & 20.1 & 14.9 & 26.8 & 25.5 & 26 & $\begin{array}{c}23.3 \pm 4.0 \\
(19.1-29.6)\end{array}$ & $\begin{array}{c}27.7 \pm 2.9 \\
(25.1-30.8)\end{array}$ \\
\hline $\mathrm{b}$ & 7.4 & 6.2 & 6.1 & 6.3 & 6.4 & 7 & $\begin{array}{l}6.8 \pm 1.1 \\
(5.5-8.0)\end{array}$ & $\begin{array}{l}8.1 \pm 1.0 \\
(7.0-8.9)\end{array}$ \\
\hline $\mathrm{c}$ & 12.5 & 12.9 & 15.2 & 14.5 & 13.6 & 17 & $\begin{array}{c}14.3 \pm 2.0 \\
(12.0-16.0)\end{array}$ & $\begin{array}{c}15.2 \pm 2.6 \\
(12.7-17.9)\end{array}$ \\
\hline$c^{\prime}$ & $?$ & 2.5 & 1.6 & 3.3 & 2.3 & 2.1 & $\begin{array}{l}2.4 \pm 0.3 \\
(2.2-2.8)\end{array}$ & $\begin{array}{l}2.4 \pm 0.3 \\
(2.1-2.7)\end{array}$ \\
\hline $\mathrm{V}(\%) / \mathrm{T}(\%)$ & $?$ & 60.7 & 64.2 & 62.3 & $?$ & $?$ & $\begin{array}{c}61.5 \pm 1.9 \\
(59.6-63.5)\end{array}$ & $\begin{array}{c}48.1 \pm 4.7 \\
(43.6-53.1)\end{array}$ \\
\hline Labial region diameter & $?$ & 16 & 18 & 12 & 17 & 17 & $\begin{array}{c}14.9 \pm 0.8 \\
(14.0-16.0)\end{array}$ & $\begin{array}{c}15.0 \pm 1.1 \\
(14.0-16.0)\end{array}$ \\
\hline Cephalic setae length & $?$ & $?$ & $?$ & 7.5 & 7.5 & 4 & $\begin{array}{l}5.5 \pm 1.7 \\
(3.5-7.5)\end{array}$ & $\begin{array}{l}7.4 \pm 0.4 \\
(7.0-7.5)\end{array}$ \\
\hline $\begin{array}{l}\text { Subdorsal cephalic setae } \\
\text { from anterior end }\end{array}$ & $?$ & $?$ & $?$ & $?$ & $?$ & 11 & $\begin{array}{l}6.6 \pm 0.6 \\
(5.5-7.0)\end{array}$ & $\begin{array}{l}7.6 \pm 0.7 \\
(7.0-8.0)\end{array}$ \\
\hline $\begin{array}{l}\text { Subventral cephalic } \\
\text { setae from anterior end }\end{array}$ & $?$ & $?$ & $?$ & $?$ & $?$ & 9 & $\begin{array}{l}6.5 \pm 0.6 \\
(5.5-7.0)\end{array}$ & $\begin{array}{l}7.1 \pm 0.4 \\
(7.0-7.5)\end{array}$ \\
\hline $\begin{array}{l}\text { Cephalic setae length / } \\
\text { labial region diam. }\end{array}$ & $?$ & $?$ & $?$ & $?$ & $?$ & $?$ & $\begin{array}{l}0.4 \pm 0.1 \\
(0.2-0.5)\end{array}$ & 0.5 \\
\hline $\begin{array}{l}\text { Anterior end of shield } \\
\text { from anterior end }\end{array}$ & $?$ & 10 & $?$ & $?$ & $?$ & $?$ & $\begin{array}{l}7.5 \pm 0.6 \\
(7.0-8.0)\end{array}$ & $\begin{array}{l}10.0 \pm 1.5 \\
(9.0-11.0)\end{array}$ \\
\hline Refractive plate length & $?$ & 25 & $?$ & $?$ & $?$ & 32 & $\begin{array}{l}25.7 \pm 1.5 \\
(23.5-27.0)\end{array}$ & $\begin{array}{c}24.5 \pm 0.5 \\
(24.0-25.0)\end{array}$ \\
\hline Refractive plate width & $?$ & 12 & $?$ & $?$ & $?$ & 12.5 & $\begin{array}{c}13.9 \pm 1.3 \\
(12.5-16.0)\end{array}$ & $\begin{array}{c}15.2 \pm 1.0 \\
(14.5-16.0)\end{array}$ \\
\hline $\begin{array}{l}\text { Anterior end of amphid } \\
\text { from anterior end }\end{array}$ & $?$ & 7 & 6 & $?$ & $?$ & 6 & $\begin{array}{l}5.5 \pm 0.7 \\
(5.0-6.0)\end{array}$ & $\begin{array}{l}6.4 \pm 1.1 \\
(5.5-7.5)\end{array}$ \\
\hline $\begin{array}{l}\text { Dorsal amphid branch } \\
\text { length }\end{array}$ & $?$ & 26 & 26 & 29 & 29 & 32 & $\begin{array}{c}23.3 \pm 2.4 \\
(21.0-25.5)\end{array}$ & $\begin{array}{c}22.5 \pm 1.1 \\
(21.5-23.5)\end{array}$ \\
\hline $\begin{array}{l}\text { Ventral amphid branch } \\
\text { length }\end{array}$ & $?$ & 23 & 24 & $?$ & $?$ & 30 & $\begin{array}{l}22.6 \pm 1.6 \\
(21.0-24.0)\end{array}$ & $\begin{array}{c}21.9 \pm 0.4 \\
(21.5-22.0)\end{array}$ \\
\hline Amphid width & $?$ & 6 & 7 & 6 & 6 & 7 & $\begin{array}{l}5.1 \pm 0.6 \\
(4.0-5.5)\end{array}$ & $\begin{array}{l}5.8 \pm 0.4 \\
(5.5-6.0)\end{array}$ \\
\hline Amphid length / width & $?$ & 4.3 & 3.7 & 4.8 & 4.8 & 4.6 & $\begin{array}{l}4.6 \pm 0.9 \\
(3.8-6.2)\end{array}$ & $\begin{array}{l}3.9 \pm 0.3 \\
(3.7-4.3)\end{array}$ \\
\hline $\begin{array}{l}\text { Anterior edge of stoma } \\
\text { from anterior end }\end{array}$ & $?$ & 1 & 1 & $?$ & $?$ & $?$ & $\begin{array}{l}1.1 \pm 0.4 \\
(1.0-1.5)\end{array}$ & $\begin{array}{l}1.4 \pm 0.7 \\
(1.0-2.0)\end{array}$ \\
\hline
\end{tabular}


Table 3 (continued).

\begin{tabular}{|c|c|c|c|c|c|c|c|c|}
\hline \multirow[b]{2}{*}{ Stoma width } & \multirow{2}{*}{$\begin{array}{c}\begin{array}{c}\text { original } \\
\text { description }\end{array} \\
?\end{array}$} & \multirow{2}{*}{$\begin{array}{c}\text { D. ovalis } \\
?\end{array}$} & \multirow{2}{*}{$\begin{array}{c}\text { D. } \\
\text { asymmetricus } \\
?\end{array}$} & \multicolumn{2}{|c|}{$\begin{array}{l}\text { D. onustus } \\
\text { sensu } \\
\text { Sergeeva } \\
(1974)\end{array}$} & \multirow{2}{*}{$\begin{array}{c}\text { data for } D . \\
\text { cuspidiboja } \\
?\end{array}$} & \multicolumn{2}{|c|}{ recent specimens } \\
\hline & & & & $?$ & $?$ & & $\begin{array}{l}5.4 \pm 0.6 \\
(5.0-6.0)\end{array}$ & $\begin{array}{l}5.3 \pm 0.4 \\
(5.0-5.5)\end{array}$ \\
\hline $\begin{array}{l}\text { Nerve ring from anterior } \\
\text { end }\end{array}$ & $?$ & $?$ & $?$ & 84 & 96 & 72 & $\begin{array}{c}90 \pm 5 \\
(84-97)\end{array}$ & $\begin{array}{l}96 \pm 10 \\
(86-104)\end{array}$ \\
\hline $\begin{array}{l}\text { Nerve ring from anterior } \\
\text { end }(\% \text { pharynx })\end{array}$ & $?$ & $?$ & $?$ & $?$ & $?$ & 41 & $\begin{array}{l}51.1 \pm 1.0 \\
(50.3-52.7)\end{array}$ & $\begin{array}{c}52.7 \pm 0.8 \\
(51.7-53.2)\end{array}$ \\
\hline $\begin{array}{l}\text { Secretory-excretory pore } \\
\text { from ant. end }\end{array}$ & $?$ & 115 & $?$ & $?$ & $?$ & 104 & $\begin{array}{c}109 \pm 3 \\
(106-112)\end{array}$ & $\begin{array}{c}110 \pm 7 \\
(103-115)\end{array}$ \\
\hline $\begin{array}{l}\text { Secr.-excr. pore from } \\
\text { ant. end ( } \% \text { pharynx })\end{array}$ & $?$ & 58.4 & $?$ & $?$ & $?$ & 60 & $\begin{array}{l}62.2 \pm 2.8 \\
(58.0-65.5)\end{array}$ & $\begin{array}{c}60.3 \pm 1.6 \\
(59.1-62.1)\end{array}$ \\
\hline Vagina or spicules length & $?$ & $?$ & $?$ & $?$ & 45 & 58 & $\begin{array}{c}18.2 \pm 1.1 \\
(17.0-19.5)\end{array}$ & $\begin{array}{c}63.8 \pm 8.2 \\
(57.0-73.0)\end{array}$ \\
\hline $\begin{array}{l}\text { Vagina / BD or spicules } \\
\text { / ABD }\end{array}$ & $?$ & $?$ & $?$ & $?$ & 1.1 & $?$ & $\begin{array}{l}0.4 \pm 0.1 \\
(0.3-0.5)\end{array}$ & $\begin{array}{l}1.6 \pm 0.3 \\
(1.3-1.8)\end{array}$ \\
\hline $\begin{array}{l}\text { Rectum or } \\
\text { gubernaculum length }\end{array}$ & $?$ & $?$ & $?$ & $?$ & $?$ & 20 & $\begin{array}{l}23.7 \pm 5.0 \\
(20.5-32.0)\end{array}$ & $\begin{array}{c}11.0 \pm 0.7 \\
(10.0-11.5)\end{array}$ \\
\hline $\begin{array}{l}\text { Rectum / ABD or } \\
\text { apophysis length }\end{array}$ & $?$ & $?$ & $?$ & $?$ & $?$ & $?$ & $\begin{array}{l}0.7 \pm 0.1 \\
(0.6-0.7)\end{array}$ & $\begin{array}{l}18.6 \pm 2.4 \\
(16.0-20.5)\end{array}$ \\
\hline $\begin{array}{l}\text { Caudal gland openings } \\
\text { from tail terminus }\end{array}$ & $?$ & $?$ & $?$ & $?$ & $?$ & 3 & $\begin{array}{l}2.0 \pm 0.6 \\
(1.0-2.0)\end{array}$ & $\begin{array}{l}1.9 \pm 0.7 \\
(1.0-2.0)\end{array}$ \\
\hline
\end{tabular}

part of tail (striation can be observed under LM but very fine and shallow and cannot be measured with confidence); longitudinal striation not observed. Somatic sensilla visible along pharyngeal region (cervical sensilla, see below) and on tail. Labial region bluntly rounded, lips fused. Refractive plates underlying cephalic cuticle around amphids present (periamphideal), extending from level of anteriormost edge of amphid posteriorly some distance behind posteriormost edge of amphid; refractive plates elongated ovoid in shape (plate edges not incised), not connected with each other on ventral and on dorsal sides. Inner labial sensilla indistinct. Outer labial sensilla papilliform, located on anterior surface of lips. Cephalic sensilla setiform, bases of dorsosublateral setae located posterior to oral opening. Cervical sensilla papilliform, arranged in four sublateral rows starting at level with middle of amphid and ending at level with posterior edge of refractive plate, one or two per row. Amphids similar in shape and size between sexes: amphidial fovea inverted U-shape with dorsal branch usually $0.5-2.0 \mu \mathrm{m}$ longer than ventral branch (in two specimens ventral branch $0.5-1.0 \mu \mathrm{m}$ longer than dorsal branch). Oral opening shifted towards dorsal side of body. Stoma barrel-shaped: cheilostom broad; gymnostom subcylindrical, with weakly cuticularised walls; stegostom short conoid, its lining uniform with lining of pharynx. Pharyngeal tubes absent. Pharynx subcylindrical, muscular, with evenly distributed myofilaments, gradually expanding towards posterior end; not subdivided in distinct sections; pharyngeal lumen uniform in thickness along entire pharynx length; valves absent. Cardia ovoid, entirely embedded into intestinal tissue. Secretoryexcretory system present; secretory-excretory pore along ventral body line opposite to $3 / 5^{\text {th }}$ of pharynx; secretory-excretory duct very short, leading from pore to ampulla; renette cell small, its body adjacent and ventral to posterior part of pharynx. Tail conoid with bluntly rounded terminus. Caudal glands opening via three separate subterminal openings, spinneret absent.

\section{Female}

Reproductive system didelphic, amphidelphic, ovary branches outstretched and symmetrical, on opposite sides of intestine. Anterior genital tube $179-269 \mu \mathrm{m}$ long, situated to either right $(n=3)$ or left $(n=2)$ of 
intestine; posterior genital tube 173-286 $\mu \mathrm{m}$ long, situated to either left $(n=3)$ or right $(n=2)$ of intestine. Vulva transverse slit, located posterior to midbody. Vagina straight, with developed sphincter muscle surrounding proximal part and distinct epiptygmata in distal part; pars refringens vaginae absent. Sacklike spermatheca present, filled with oval spermatozoa in fertilized specimens. Rectum short.

\section{Male}

Reproductive system diorchic, testes opposed; anterior testis 145-314 $\mu \mathrm{m}$ long, outstretched and posterior testis 117-207 $\mu \mathrm{m}$ long, reflexed. Spicules paired and symmetrical, strongly curved, with weakly defined elongated manubrium and shaft, cylindrical along most of its length. Gubernaculum plate-like, with pair of strong, closely set caudal apophyses variable in shape (Fig. 7C-E). Caudal setae present, several ventrosublateral and dorsosublateral pairs arranged in rows along entire tail length (often difficult to observe).

\section{Remarks}

The original description of this species by Steiner (1916) is rather short, with a few measurements given. The present population has a shorter pharynx and tail compared to the type specimen, but in all other respects both match very well, including the position of the oral opening and the shape of the underlying refractive plates.

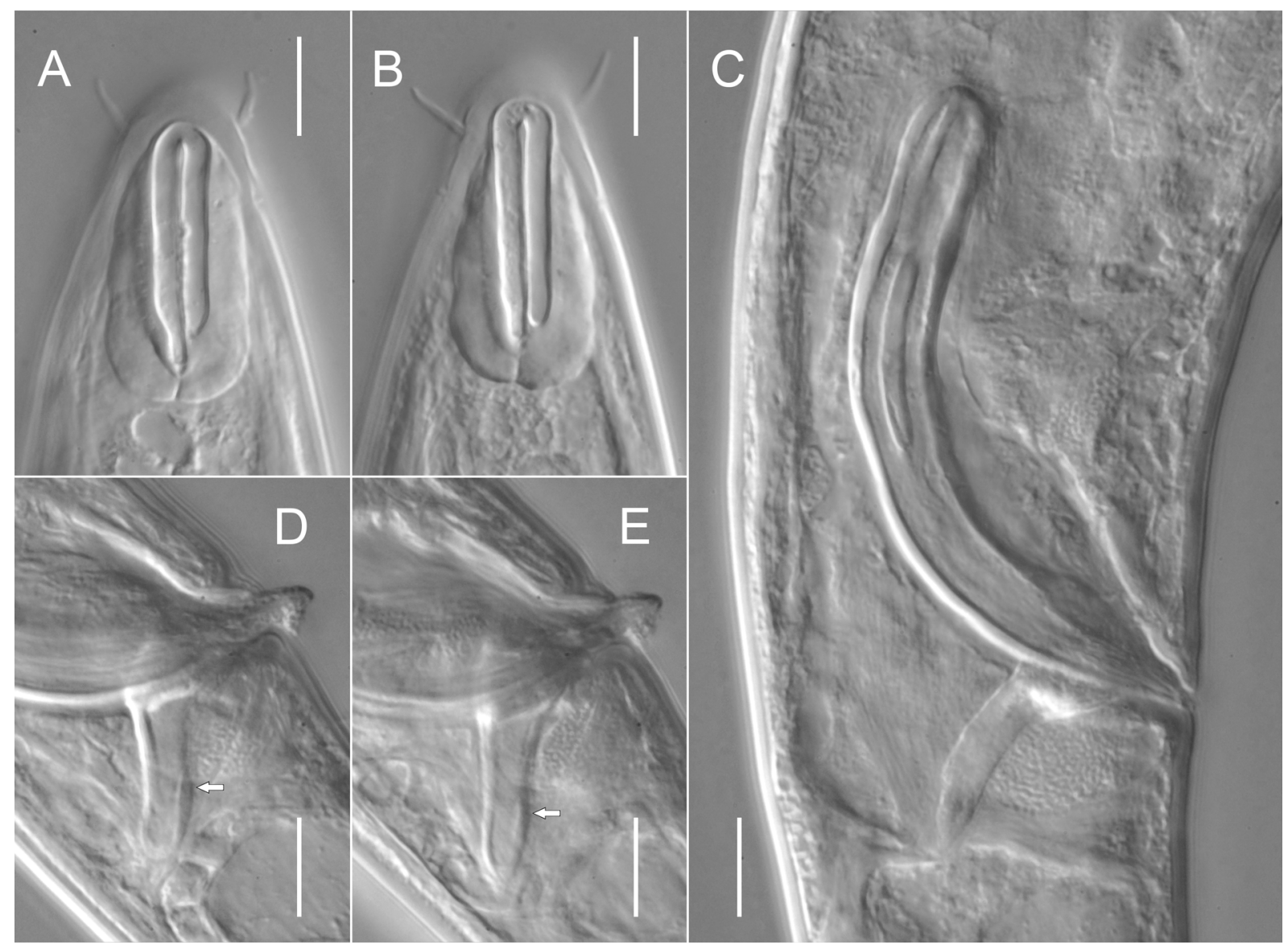

Fig. 7. Neodiplopeltula barentsi (Steiner, 1916) gen. et comb. nov. (SMNH-169266 and SMNH-169268). A. Female anterior end, lateral view. B. Male anterior end, lateral view. C. Spicules and gubernaculum. D-E. Variability in the shape of thegubernaculum apophyses in the same specimen (arrows). Scale bars $=10 \mu \mathrm{m}$. 
Neodiplopeltula onusta (Wieser, 1956) gen. et comb. nov.

Figs 8-10; Table 4

Diagnosis (based on combined data)

Neodiplopeltula onusta gen. et comb. nov. is characterised by a $0.99-1.48 \mathrm{~mm}$ long body; refractive plates underlying cephalic cuticle around amphids present and joined, 32.5-48 $\mu \mathrm{m}$ long and 18.5-24 $\mu \mathrm{m}$ wide; cephalic setae 3-6.5 $\mu \mathrm{m}$ long; amphidial fovea 32-44 $\mu \mathrm{m}$ long and 5-8 $\mu \mathrm{m}$ wide; oral opening
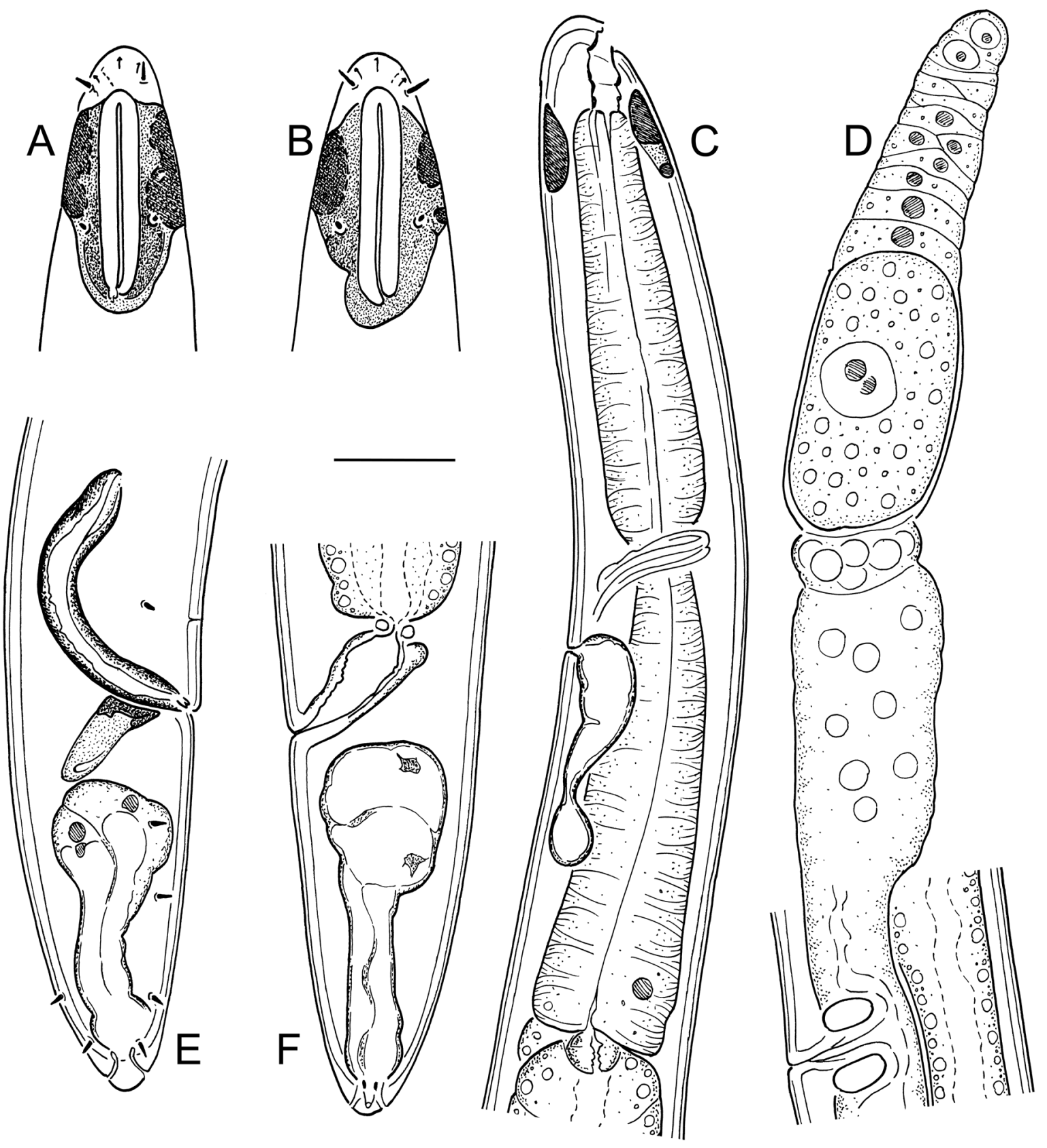

Fig. 8. Neodiplopeltula onusta (Wieser, 1956) gen. et comb. nov. (SMNH-169282). A. Male anterior end, surface view. B. Female anterior end, surface view. C. Female pharyngeal region, optical median section. D. Anterior part of female reproductive system. E. Male tail. F. Female tail. Scale bar $=20 \mu \mathrm{m}$. 
Table 4 (continued on next page). Measurements (in $\mu \mathrm{m}$ ) of Neodiplopeltula onusta (Wieser, 1956) gen. et comb. nov.

\begin{tabular}{|c|c|c|c|c|c|}
\hline & \multirow{2}{*}{$\begin{array}{c}\begin{array}{c}\text { original } \\
\text { description }\end{array} \\
q\end{array}$} & \multicolumn{2}{|c|}{ data for Diplopeltula tchesunovi } & \multicolumn{2}{|c|}{ recent specimens } \\
\hline & & 4 우우 & $3 \widehat{0}$ & 4 우 & 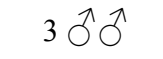 \\
\hline Body length & 990 & $1348-1481$ & $1003-1323$ & $\begin{array}{l}1064 \pm 104 \\
(947-1199)\end{array}$ & $\begin{array}{l}1056 \pm 50 \\
(999-1094)\end{array}$ \\
\hline Body diameter (BD) & $?$ & $65-88$ & $48-58$ & $\begin{array}{c}47 \pm 9 \\
(38-55)\end{array}$ & $\begin{array}{c}46 \pm 7 \\
(39-53)\end{array}$ \\
\hline Pharynx length & $?$ & $201-223$ & $172-212$ & $\begin{array}{c}170 \pm 17 \\
(149-187)\end{array}$ & $\begin{array}{c}174 \pm 15 \\
(159-189)\end{array}$ \\
\hline Tail length & $?$ & $75-77$ & $65-81$ & $\begin{array}{c}58 \pm 7 \\
(51-68)\end{array}$ & $\begin{array}{l}74 \pm 12 \\
(65-88)\end{array}$ \\
\hline Anal body diameter (ABD) & $?$ & $44-52$ & $37-41$ & $\begin{array}{c}34 \pm 3 \\
(32-37)\end{array}$ & $\begin{array}{c}37 \pm 3 \\
(34-39)\end{array}$ \\
\hline $\mathrm{a}$ & 26 & $17-23$ & $21-23$ & $\begin{array}{c}23.6 \pm 5.9 \\
(19.3-31.9)\end{array}$ & $\begin{array}{c}23.4 \pm 4.4 \\
(20.5-28.4)\end{array}$ \\
\hline $\mathrm{b}$ & 5.1 & $6.3-7.3$ & $5.9-6.3$ & $\begin{array}{l}6.3 \pm 0.8 \\
(5.3-7.2)\end{array}$ & $\begin{array}{l}6.1 \pm 0.3 \\
(5.8-6.3)\end{array}$ \\
\hline $\mathrm{c}$ & 14.2 & $17.5-19.2$ & $12.5-17.4$ & $\begin{array}{c}18.7 \pm 3.1 \\
(15.2-21.4)\end{array}$ & $\begin{array}{c}14.6 \pm 2.8 \\
(11.4-16.9)\end{array}$ \\
\hline$c^{\prime}$ & 1.7 & $?$ & $?$ & $\begin{array}{l}1.7 \pm 0.3 \\
(1.4-2.2)\end{array}$ & $\begin{array}{l}2.0 \pm 0.2 \\
(1.8-2.3)\end{array}$ \\
\hline $\mathrm{V}(\%) / \mathrm{T}(\%)$ & 65.4 & $65.1-67.6$ & $?$ & $\begin{array}{c}66.1 \pm 1.5 \\
(64.7-68.0)\end{array}$ & $\begin{array}{c}41.0 \pm 7.9 \\
(35.4-46.6)\end{array}$ \\
\hline Labial region diameter & 15 & $12-16.5$ & $14-15$ & $\begin{array}{l}11.2 \pm 1.7 \\
(9.0-13.0)\end{array}$ & $\begin{array}{c}12.0 \pm 0.8 \\
(11.0-12.5)\end{array}$ \\
\hline Cephalic setae length & 6.5 & $3.5-5.4$ & $3.5-4.5$ & $\begin{array}{l}4.0 \pm 1.2 \\
(3.0-5.5)\end{array}$ & $\begin{array}{l}3.9 \pm 0.8 \\
(3.5-5.0)\end{array}$ \\
\hline $\begin{array}{l}\text { Subdorsal cephalic setae from } \\
\text { anterior end }\end{array}$ & 8 & $?$ & $?$ & $\begin{array}{l}6.4 \pm 0.7 \\
(5.5-7.0)\end{array}$ & $\begin{array}{l}7.4 \pm 1.1 \\
(6.0-8.0)\end{array}$ \\
\hline $\begin{array}{l}\text { Subventral cephalic setae from } \\
\text { anterior end }\end{array}$ & $?$ & $?$ & ? & $\begin{array}{l}5.9 \pm 0.7 \\
(5.0-6.0)\end{array}$ & $\begin{array}{l}6.2 \pm 0.7 \\
(5.5-7.0)\end{array}$ \\
\hline $\begin{array}{l}\text { Cephalic setae length / labial } \\
\text { region diam. }\end{array}$ & $?$ & $?$ & $?$ & $\begin{array}{l}0.4 \pm 0.1 \\
(0.3-0.5)\end{array}$ & $\begin{array}{l}0.3 \pm 0.1 \\
(0.3-0.4)\end{array}$ \\
\hline $\begin{array}{l}\text { Anterior end of shield from } \\
\text { anterior end }\end{array}$ & ? & $?$ & ? & $\begin{array}{c}9.2 \pm 0.4 \\
(9.0-10.0)\end{array}$ & $\begin{array}{c}10.1 \pm 0.4 \\
(10.0-10.5)\end{array}$ \\
\hline Refractive plate length & $?$ & $45-48$ & $41-47$ & $\begin{array}{c}36.1 \pm 3.2 \\
(32.5-38.0)\end{array}$ & $\begin{array}{c}35.0 \pm 1.1 \\
(34.0-36.0)\end{array}$ \\
\hline Refractive plate width & $?$ & $20-24$ & $19-20$ & $\begin{array}{c}21.6 \pm 1.5 \\
(19.0-23.0)\end{array}$ & $\begin{array}{c}20.0 \pm 1.4 \\
(18.5-21.5)\end{array}$ \\
\hline $\begin{array}{l}\text { Anterior end of amphid from } \\
\text { anterior end }\end{array}$ & $?$ & $4-7$ & $5-7$ & $\begin{array}{l}5.9 \pm 0.9 \\
(5.0-7.0)\end{array}$ & $\begin{array}{l}7.4 \pm 0.8 \\
(7.0-8.0)\end{array}$ \\
\hline Dorsal amphid branch length & 40 & $41-44$ & $36-43$ & $\begin{array}{c}36.2 \pm 2.6 \\
(32.5-38.0)\end{array}$ & $\begin{array}{c}35.9 \pm 0.7 \\
(35.0-36.5)\end{array}$ \\
\hline Ventral amphid branch length & 40 & $39-43$ & $34-41$ & $\begin{array}{c}35.0 \pm 2.5 \\
(32.0-37.0)\end{array}$ & $\begin{array}{c}34.3 \pm 1.1 \\
(33.0-35.0)\end{array}$ \\
\hline Amphid width & 8 & $5-7$ & $5-6$ & $\begin{array}{l}6.6 \pm 0.9 \\
(5.5-7.5)\end{array}$ & $\begin{array}{l}6.7 \pm 0.4 \\
(6.0-7.0)\end{array}$ \\
\hline
\end{tabular}


Table 4 (continued).

\begin{tabular}{|c|c|c|c|c|c|}
\hline \multirow[b]{2}{*}{ Amphid length / width } & \multirow{2}{*}{$\begin{array}{c}\begin{array}{c}\text { original } \\
\text { description }\end{array} \\
? ?\end{array}$} & \multicolumn{2}{|c|}{ data for Diplopeltula tchesunovi } & \multicolumn{2}{|c|}{ recent specimens } \\
\hline & & $?$ & $?$ & $\begin{array}{l}5.6 \pm 0.7 \\
(5.0-6.6)\end{array}$ & $\begin{array}{l}5.4 \pm 0.2 \\
(5.2-5.7)\end{array}$ \\
\hline $\begin{array}{l}\text { Anterior edge of stoma from } \\
\text { anterior end }\end{array}$ & ? & 5 & 5 & $\begin{array}{l}2.8 \pm 0.8 \\
(2.0-3.5)\end{array}$ & $\begin{array}{l}3.7 \pm 1.1 \\
(3.0-5.0)\end{array}$ \\
\hline Stoma length & $?$ & $?$ & $?$ & $\begin{array}{c}13.6 \pm 0.7 \\
(13.0-14.5)\end{array}$ & $\begin{array}{c}13.6 \pm 0.4 \\
(13.0-14.0)\end{array}$ \\
\hline Stoma width & $?$ & $?$ & $?$ & $\begin{array}{l}4.8 \pm 0.6 \\
(4.0-5.5)\end{array}$ & 5.0 \\
\hline Nerve ring from anterior end & ? & $96-106$ & $90-103$ & $\begin{array}{l}92 \pm 6 \\
(84-96)\end{array}$ & $\begin{array}{c}95 \pm 6 \\
(91-102)\end{array}$ \\
\hline $\begin{array}{l}\text { Nerve ring from anterior end } \\
(\% \text { pharynx) }\end{array}$ & ? & ? & ? & $\begin{array}{c}54.4 \pm 2.9 \\
(50.5-56.8)\end{array}$ & $\begin{array}{c}54.3 \pm 2.6 \\
(52.0-57.1)\end{array}$ \\
\hline $\begin{array}{l}\text { Secretory-excretory pore from ant. } \\
\text { end }\end{array}$ & 115 & $110-125$ & $85-87$ & $\begin{array}{c}104 \pm 8 \\
(95-112)\end{array}$ & $\begin{array}{c}107 \pm 4 \\
(103-112)\end{array}$ \\
\hline $\begin{array}{l}\text { Secr.-excr. pore from ant. end } \\
(\% \text { pharynx) }\end{array}$ & 66 & $?$ & $?$ & $\begin{array}{c}61.1 \pm 2.9 \\
(57.0-63.5)\end{array}$ & $\begin{array}{c}61.7 \pm 2.9 \\
(59.3-64.8)\end{array}$ \\
\hline Vagina or spicules length & $?$ & $35-40$ & $62-72$ & $\begin{array}{c}19.7 \pm 2.7 \\
(16.5-23.0)\end{array}$ & $\begin{array}{l}63.7 \pm 11.8 \\
(56.5-77.0)\end{array}$ \\
\hline Vagina / BD or spicules / ABD & ? & ? & $1.3-1.5$ & $\begin{array}{l}0.4 \pm 0.1 \\
(0.3-0.6)\end{array}$ & $\begin{array}{l}1.7 \pm 0.3 \\
(1.5-2.0)\end{array}$ \\
\hline Rectum or gubernaculum length & $?$ & $?$ & $15-20$ & $\begin{array}{c}24.1 \pm 3.6 \\
(21.0-28.0)\end{array}$ & $\begin{array}{c}10.0 \pm 0.5 \\
(10.0-10.5)\end{array}$ \\
\hline Rectum / ABD or apophysis length & $?$ & ? & $?$ & $\begin{array}{l}0.7 \pm 0.1 \\
(0.6-0.9)\end{array}$ & $\begin{array}{c}16.1 \pm 1.1 \\
(15.0-17.0)\end{array}$ \\
\hline $\begin{array}{l}\text { Caudal gland openings from tail } \\
\text { terminus }\end{array}$ & ? & ? & $?$ & terminal & terminal \\
\hline
\end{tabular}

2-5 from anterior end, at level with cephalic setae bases; secretory-excretory pore located opposite to posterior part of pharynx; tail 1.4-2.3 anal body diameters long; vagina with epiptygmata; spicules $52-77 \mu \mathrm{m}$ long.

\section{Material examined}

SWEDEN: 1 q, Skagerrak, 58 $19^{\prime} 15.6^{\prime \prime}-20.9^{\prime \prime} \mathrm{N}, 10^{\circ} 29^{\prime} 33.5^{\prime \prime}-34.0^{\prime \prime}$ E, soft bottom at a deep of 352-374 m, 10 Sep. 2012, "Inventering Bratten" leg. (SMNH-169284); 1 ภ, Skagerrak, 58 23'00.8"$22^{\prime} 00.8^{\prime \prime} \mathrm{N}, 10^{\circ} 20^{\prime} 28.8^{\prime \prime}-38.3^{\prime \prime} \mathrm{E}$, soft bottom at a deep of 390-428 m, 10 Sep. 2012, "Inventering

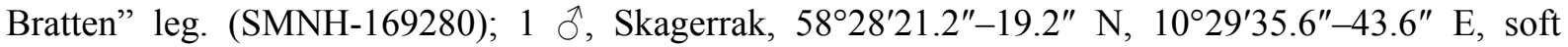
bottom at a deep of 248-316 m, 11 Sep. 2012, "Inventering Bratten" leg. (SMNH-169281); 3 우으,

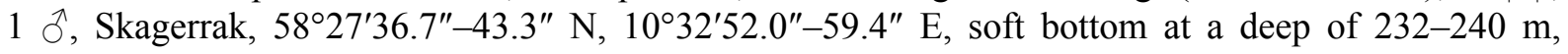
12 Sep. 2012, "Inventering Bratten" leg. (SMNH-169282 - SMNH-169283).

\section{Description}

\section{Adult}

Body cylindrical, posteriorly tapering in tail region, straight or weakly ventrally curved upon fixation. Cuticle finely transversely striated along entire body, except for visually smooth labial region and terminal part of tail (striation can be observed under LM but very fine and shallow and cannot be measured with 
confidence); longitudinal striation not observed. Somatic sensilla visible along pharyngeal region (cervical setae, see below) and on tail. Labial region bluntly rounded, lips fused. Refractive plates underlying cephalic cuticle around amphids present (periamphideal), extending from level of anteriormost edge of amphid posteriorly some distance behind posteriormost edge of amphid; refractive plates connected with each other on ventral and on dorsal sides. Inner labial sensilla indistinct. Outer labial sensilla papilliform, located on anterior surface of lips. Cephalic sensilla setiform, bases of dorsosublateral setae located at level with oral opening. Cervical sensilla papilliform, arranged in four sublateral rows at level with amphid, one or two per row. Amphids similar in shape and size between sexes: amphidial fovea inverted U-shape with dorsal branch 1.0-2.0 $\mu \mathrm{m}$ longer than ventral branch. Oral opening shifted towards dorsal side of body. Stoma subcylindrical: cheilostom broad; gymnostom barrel-shaped, with weakly cuticularised walls; stegostom short conoid, its lining uniform with lining of pharynx. Pharyngeal tubes absent. Pharynx subcylindrical, muscular, with evenly distributed myofilaments, gradually expanding towards posterior end; not subdivided in distinct sections; pharyngeal lumen uniform in thickness along

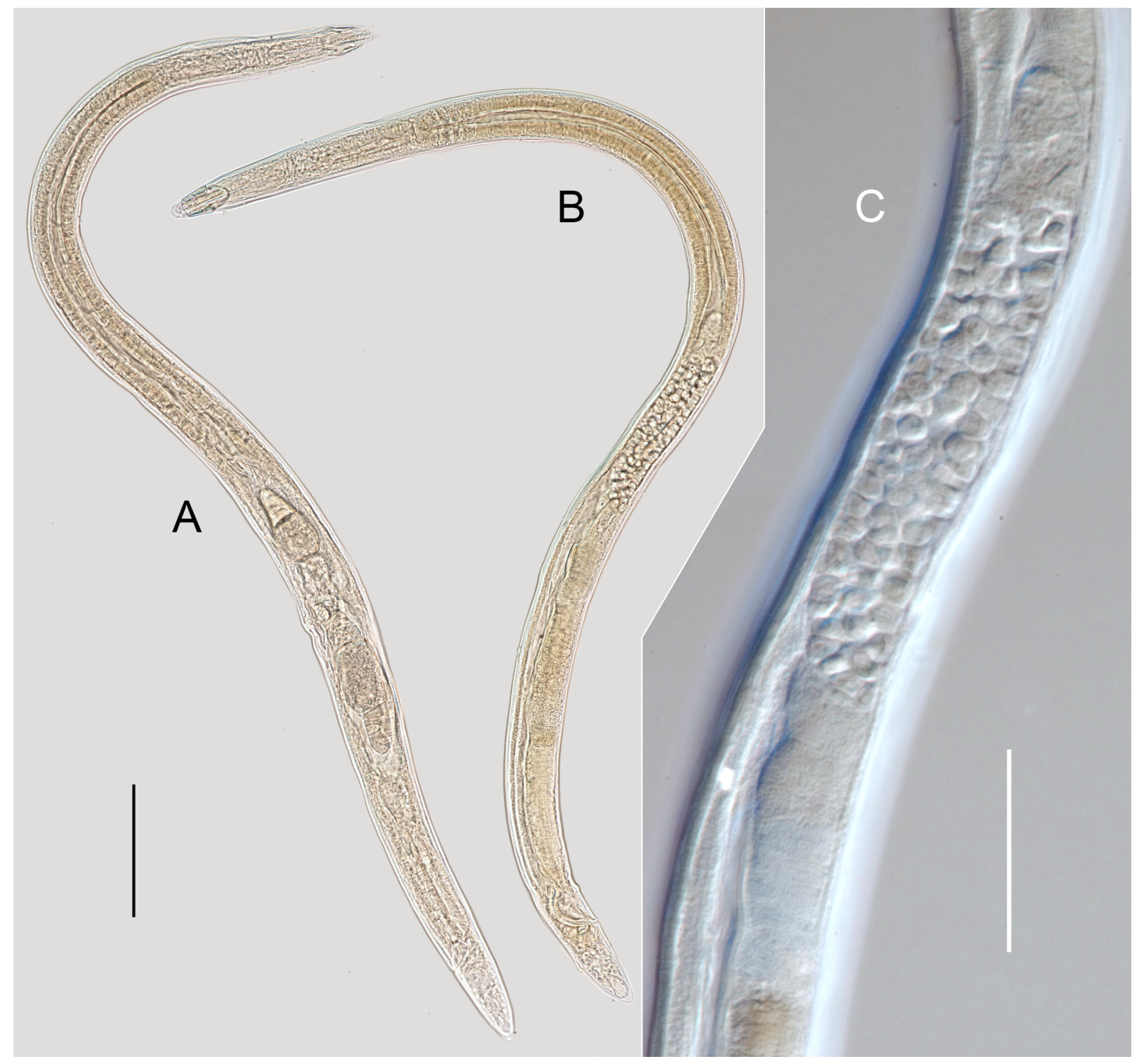

Fig. 9. Neodiplopeltula onusta (Wieser, 1956) gen. et comb. nov. (SMNH-169282). A. + , entire specimen. B. $\widehat{\partial}$, entire specimen. C. Testes. Scale bars: A-B $=100 \mu \mathrm{m} ; \mathrm{C}=50 \mu \mathrm{m}$. 
entire pharynx length; valves absent. Secretory-excretory system present; secretory-excretory pore located along ventral body line opposite to $3 / 5^{\text {th }}$ of pharynx; secretory-excretory duct very short, leading from pore to ampulla; renette cell small, its body adjacent and ventral to posterior part of pharynx. Tail conoid with bluntly rounded terminus. Caudal glands opening via three separate subterminal openings, spinneret absent.

\section{Female}

Reproductive system didelphic, amphidelphic, ovary branches outstretched and symmetrical, on opposite sides of intestine. Anterior genital tube 109-180 $\mu \mathrm{m}$ long, situated to either right $(\mathrm{n}=3)$ or left $(n=1)$ of intestine; posterior genital tube 109-145 $\mu \mathrm{m}$ long, situated to either left $(n=3)$ or right $(\mathrm{n}=1)$ of intestine. Vulva transverse slit, located posterior to midbody. Vagina straight, with developed sphincter muscle surrounding proximal part and distinct epiptygmata in distal part; pars refringens vaginae absent. Sack-like spermatheca present, filled with oval spermatozoa in fertilized specimens. Rectum short.

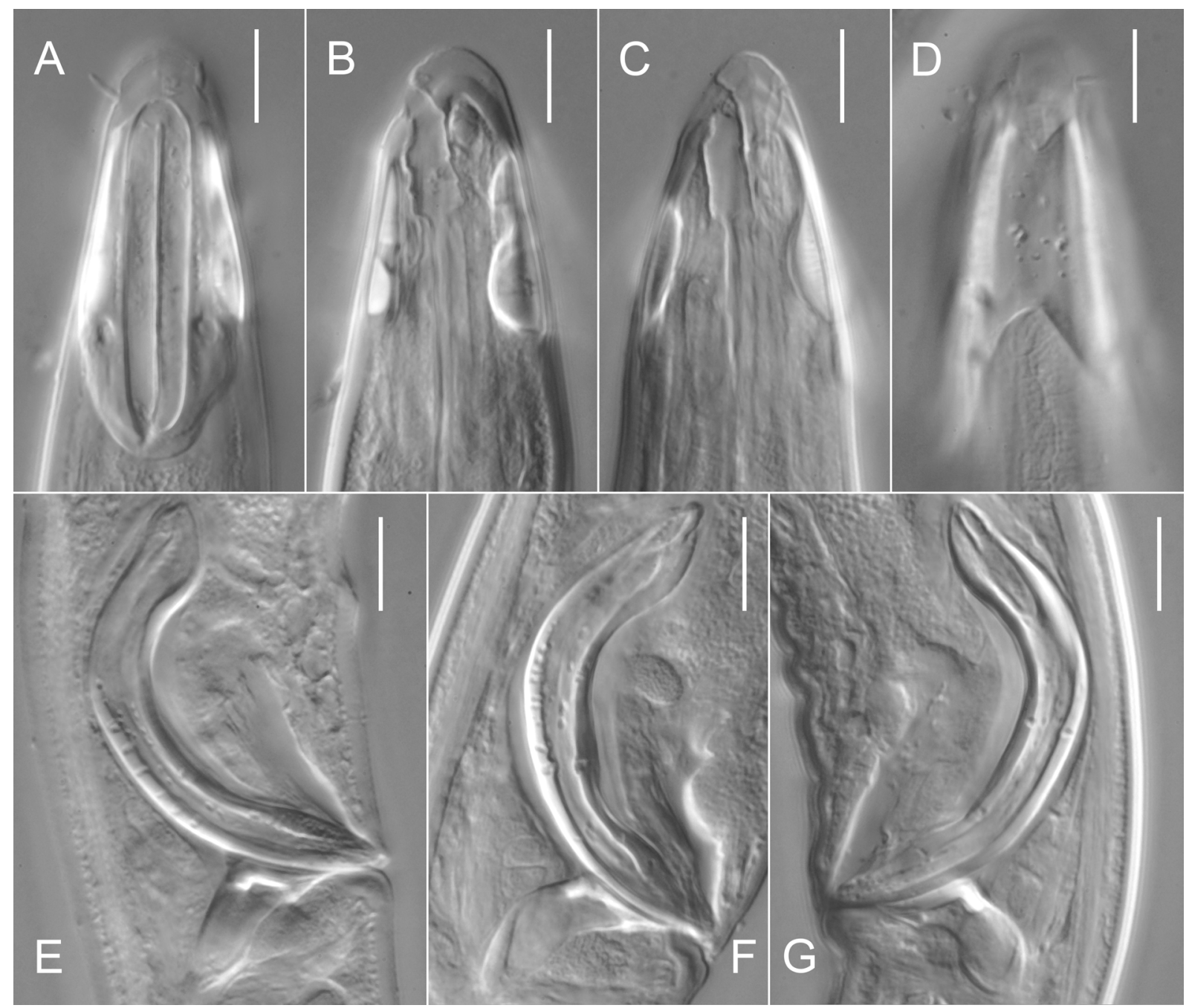

Fig. 10. Neodiplopeltula onusta (Wieser, 1956) gen. et comb. nov. (SMNH-169282). A. Male anterior end, lateral view. B. Male stoma, lateral view. C. Female stoma, lateral view. D. Male anterior end, dorsal view. E-G. Variability in the shape of spicules and gubernaculum in different specimens. Scale bars $=10 \mu \mathrm{m}$. 


\section{Male}

Reproductive system diorchic, testes opposed; anterior testis outstretched and posterior testis reflexed (poorly discernible in most specimens due to highly granular and dark content of overlapping intestine and cannot be measured). Spicules paired and symmetrical, strongly curved, with elongated ovoid manubrium and subcylindrical shaft. Gubernaculum plate-like, with pair of strong closely set caudal apophyses variable in shape (Fig. 10E-G). Caudal setae present, several ventrosublateral and dorsosublateral pairs arranged in rows along entire tail length.

\section{Remarks}

The single female used by Wieser (1956) to describe this species has a slightly broader labial region and longer cephalic setae than the population from Sweden, but the specimens described under the name Diplopeltula tchesunovi fill this gap. The position of the oral opening is at level with the cephalic setae bases and the shape of the refractive plates underlying the cephalic cuticle around the amphids (joined along the dorsal and ventral body sides) in both type female and recent specimens are identical and confirm the conspecificity.

Neodiplopeltula bathmanni (Jensen, 1991) gen. et comb. nov. Figs 11-13; Table 5

\section{Diagnosis (based on combined data)}

Neodiplopeltula bathmanni gen. et comb. nov. is characterised by a $0.85-2.08 \mathrm{~mm}$ long body; refractive plates underlying cephalic cuticle around amphids present, not joined, 19-29 $\mu \mathrm{m}$ long and 12-16.5 $\mu \mathrm{m}$ wide; cephalic sensilla 2-3.5 $\mu \mathrm{m}$ long; amphidial fovea 20-32 $\mu \mathrm{m}$ long and 4-7 $\mu \mathrm{m}$ wide; oral opening 6-16 $\mu \mathrm{m}$ from anterior end, at level with or posterior to cephalic setae bases; secretory-excretory pore located opposite to posterior part of pharynx; tail 1.5-2.2 anal body diameters long; vagina with epiptygmata; spicules 52-66 $\mu \mathrm{m}$ long.

\section{Material examined}

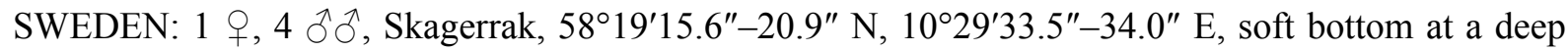
of 352-374 m, 10 Sep. 2012, "Inventering Bratten" leg. (SMNH-169274 and SMNH-169284); 1 , Skagerrak, 58 $22^{\prime} 17.8^{\prime \prime}-19.4^{\prime \prime} \mathrm{N}, 10^{\circ} 23^{\prime} 50.8^{\prime \prime}-24^{\prime} 03.2^{\prime \prime}$ E, soft bottom at a deep of 351-387 m, 10 Sep. 2012, "Inventering Bratten" leg. (SMNH-169275); 1 ㅇ, 1 ภ’, Skagerrak, 58 34'21.3"-16.6" N, $10^{\circ} 38^{\prime} 11.2^{\prime \prime}-29.4^{\prime \prime}$ E, soft bottom at a deep of 139-153 m, 12 Sep. 2012, "Inventering Bratten" leg. (SMNH-169277).

\section{Description}

\section{Adult}

Body cylindrical, posteriorly tapering in tail region, straight or weakly ventrally curved upon fixation. Cuticle finely transversely striated along entire body, except for visually smooth labial region and terminal part of tail (striation can be observed under LM but very fine and shallow and cannot be measured with confidence); longitudinal striation not observed. Somatic sensilla visible along pharyngeal region (cervical setae, see below) and on tail. Labial region bluntly rounded, lips fused. Refractive plates underlying cephalic cuticle around amphids present (periamphideal), extending from level of anteriormost edge of amphid posteriorly some distance behind the posteriormost edge of amphid; refractive plates are elongated ovoid in shape (plate edges not incised), not connected with each other on ventral and on dorsal sides. Inner labial sensilla indistinct. Outer labial sensilla papilliform, located on anterior surface of lips. Cephalic sensilla setiform, bases of dorsosublateral setae located at level with or anterior to oral opening. Cervical sensilla absent; small papilliform somatic sensilla visible posterior to amphid and refractive plate. Amphids similar in shape and size between sexes: amphidial 

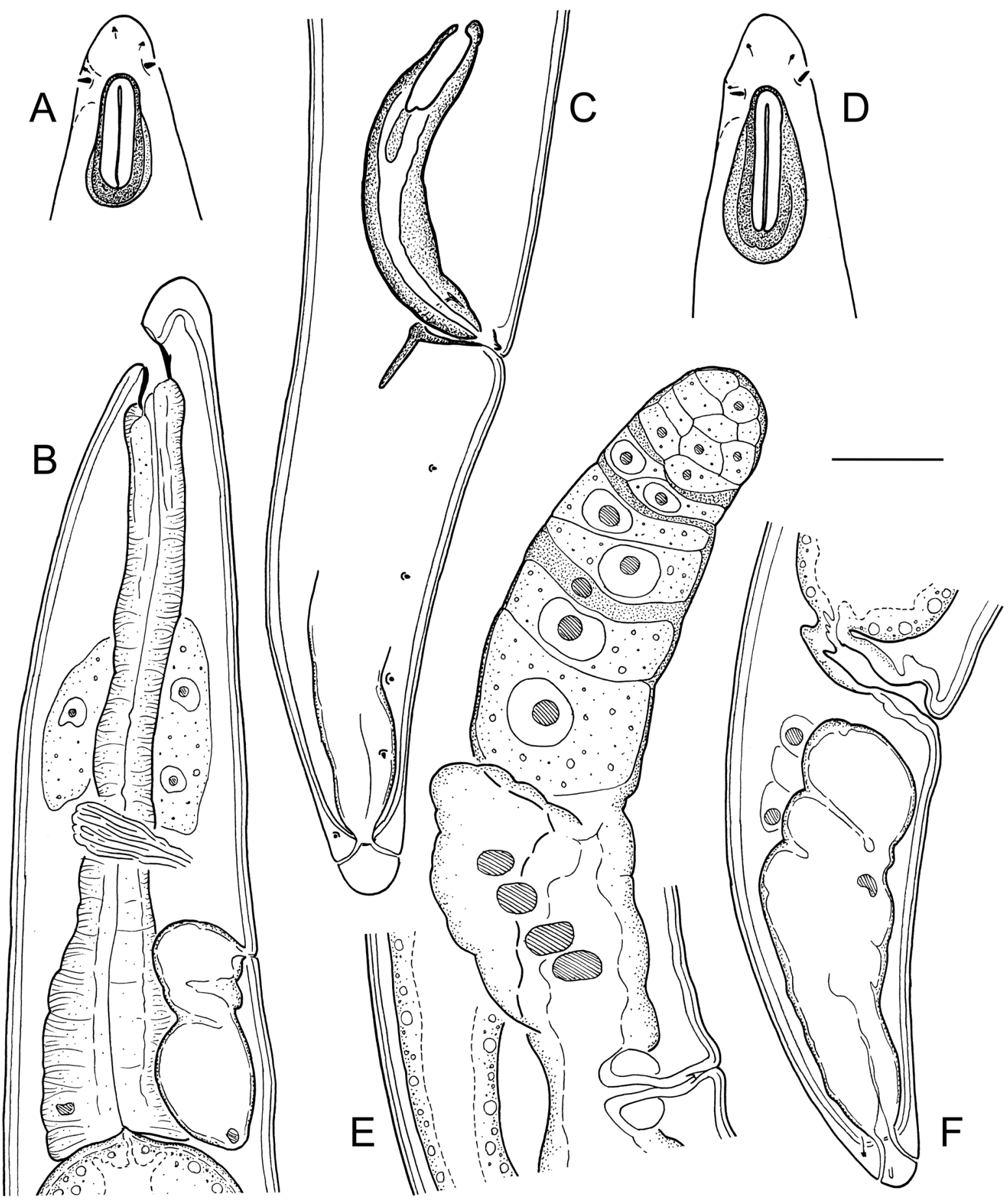

Fig. 11. Neodiplopeltula bathmanni (Jensen, 1991) gen. et comb. nov. (ð SMNH-169274 and $q$ SMNH-169277). A. Female anterior end, surface view. B. Female pharyngeal region, optical median section. C. Male tail. D. Male anterior end, surface view. E. Anterior part of female reproductive system. F. Female tail. Scale bar $=20 \mu \mathrm{m}$. 
fovea inverted U-shape branches unequal in length with either dorsal or ventral branch 1.0-2.0 $\mu \mathrm{m}$ longer than opposite branch. Oral opening shifted towards dorsal side of body. Stoma subcylindrical: cheilostom broad; gymnostom barrel-shaped, with weakly cuticularised walls; stegostom short conoid, its lining uniform with lining of pharynx. Pharyngeal tubes absent. Pharynx subcylindrical, muscular, with evenly distributed myofilaments, gradually expanding towards posterior end; not subdivided in distinct sections; pharyngeal lumen uniform in thickness along entire pharynx length; valves absent. Secretory-excretory system present; secretory-excretory pore located along ventral body line opposite to $4 / 5^{\text {th }}$ of pharynx; secretory-excretory duct very short, leading from pore to ampulla; renette cell small, its body adjacent and ventral to posterior part of pharynx. Tail conoid with bluntly rounded terminus. Caudal glands opening via three separate subterminal openings, spinneret absent.

\section{Female}

Reproductive system didelphic, amphidelphic, ovary branches outstretched and symmetrical, on opposite sides of intestine. Anterior genital tube $97-242 \mu \mathrm{m}$ long, situated to either right $(\mathrm{n}=2)$ or left $(\mathrm{n}=1)$ of

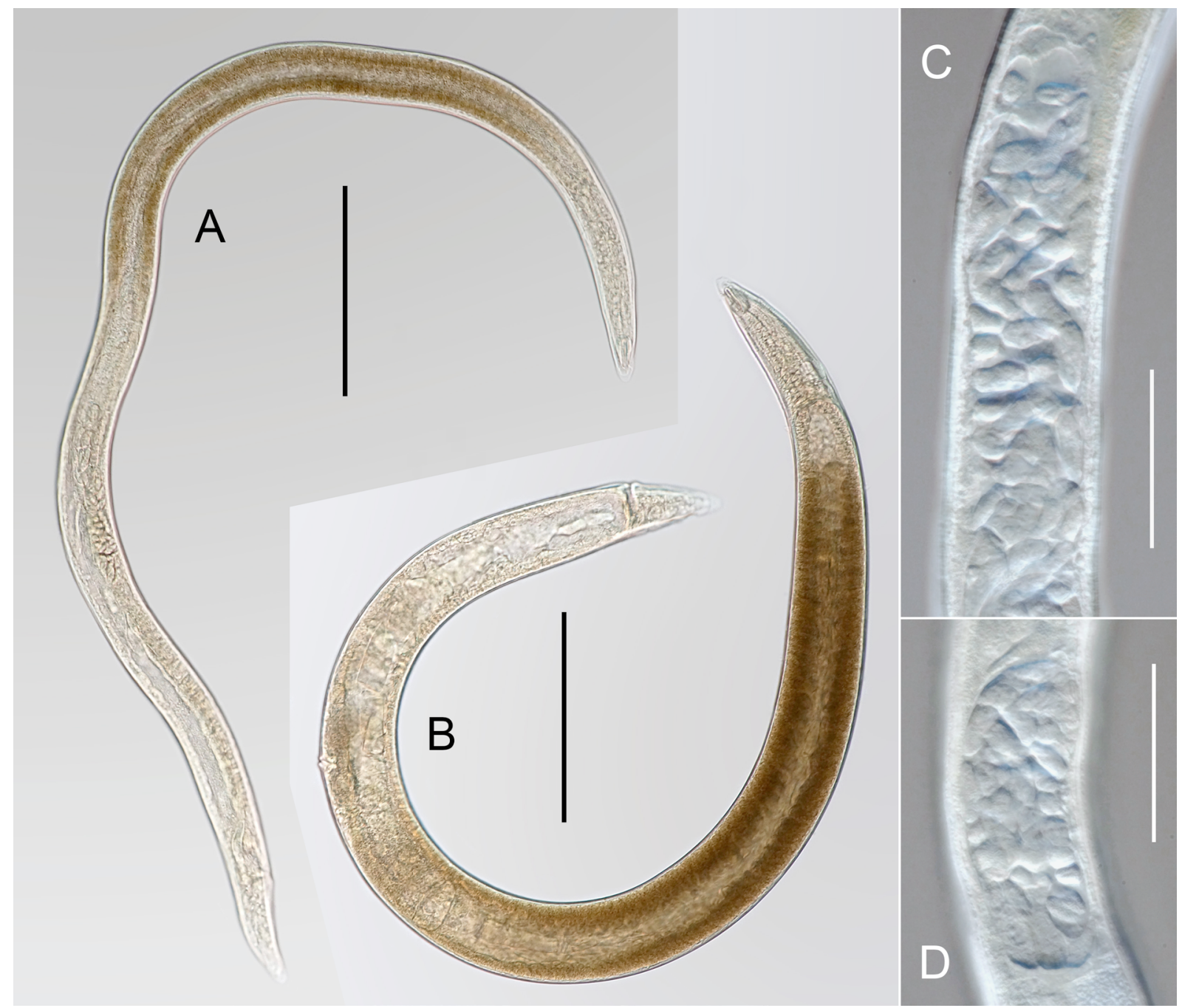

Fig. 12. Neodiplopeltula bathmanni (Jensen, 1991) gen. et comb. nov. (ð SMNH-169274 and $q$ SMNH169277). A. $\widehat{\delta}$, entire specimen. B. ${ }^{+}$, entire specimen. C. Anterior testis. D. Posterior testis. Scale bars: $\mathrm{A}-\mathrm{B}=100 \mu \mathrm{m}: \mathrm{C}-\mathrm{D}=50 \mu \mathrm{m}$. 
intestine; posterior genital tube 103-245 $\mu \mathrm{m}$ long situated to either left $(n=2)$ or right $(n=1)$ of intestine. Vulva transverse slit, located posterior to midbody. Vagina straight, with developed sphincter muscle surrounding proximal part and distinct epiptygmata in distal part; pars refringens vaginae absent. Sacklike spermatheca present, filled with oval spermatozoa in fertilized specimens. Rectum short.

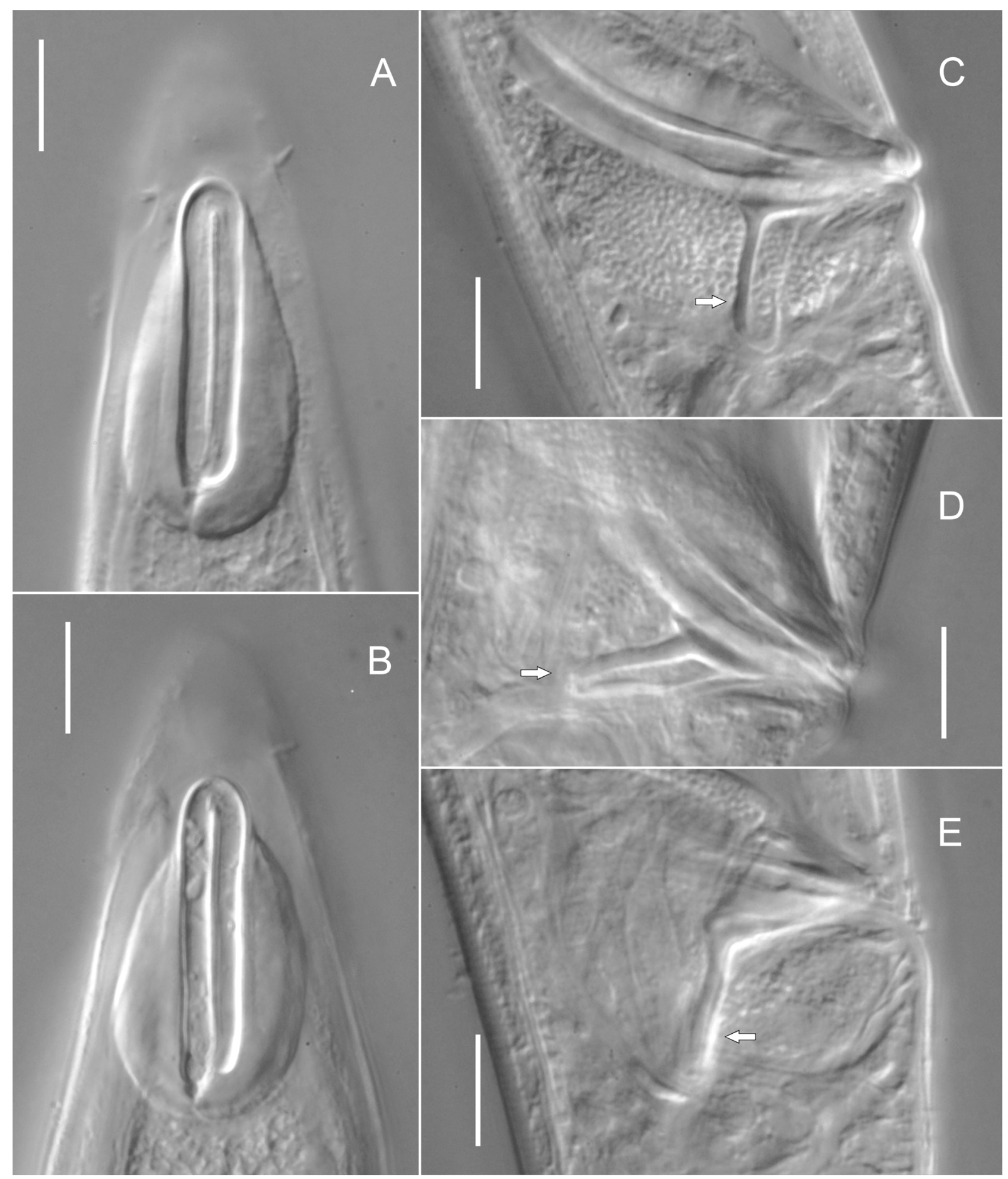

Fig. 13. Neodiplopeltula bathmanni (Jensen, 1991) gen. et comb. nov. (SMNH-169274 and SMNH169277). A. Male anterior end, lateral view. B. Female anterior end, lateral view. C-E. Variability in the shape of gubernaculum apophyses in different specimens (arrows). Scale bars $=10 \mu \mathrm{m}$. 
Table 5 (continued on next page). Measurements (in $\mu \mathrm{m}$ ) of Neodiplopeltula bathmanni (Jensen, 1991) gen. et comb. nov.

\begin{tabular}{|c|c|c|c|c|c|c|c|}
\hline & \multicolumn{2}{|c|}{ original description } & \multirow{2}{*}{$\begin{array}{c}\begin{array}{c}\text { data for } \\
\text { D. cuspidiboja }\end{array} \\
1 \uparrow\end{array}$} & \multicolumn{2}{|c|}{$\begin{array}{l}\text { D. incisa sensu } \\
\text { Vitiello, } 1972\end{array}$} & \multicolumn{2}{|c|}{ recent specimens } \\
\hline & 2 우우 & $20^{\lambda} 0^{\lambda}$ & & q & $\sigma^{\lambda}$ & 3 우우 & $50^{\lambda} 0^{\lambda}$ \\
\hline Body length & $1300-1420$ & $1150-1240$ & 2076 & 853 & 1138 & $\begin{array}{c}1467 \pm 92 \\
(1366-1546)\end{array}$ & $\begin{array}{c}1442 \pm 193 \\
(1270-1673)\end{array}$ \\
\hline Body diameter (BD) & $52-57$ & $43-47$ & 98 & $?$ & $?$ & $\begin{array}{l}71 \pm 10 \\
(60-78)\end{array}$ & $\begin{array}{c}51 \pm 8 \\
(43-60)\end{array}$ \\
\hline Pharynx length & $150-189$ & $154-170$ & 165 & $?$ & $?$ & $\begin{array}{c}154 \pm 5 \\
(148-157)\end{array}$ & $\begin{array}{c}151 \pm 14 \\
(139-171)\end{array}$ \\
\hline Tail length & $82-88$ & $77-91$ & 98 & 60 & 72 & $\begin{array}{c}84 \pm 4 \\
(81-89)\end{array}$ & $\begin{array}{c}84 \pm 8 \\
(76-96)\end{array}$ \\
\hline $\begin{array}{l}\text { Anal body diameter } \\
\text { (ABD) }\end{array}$ & 40 & 43 & 67 & $?$ & $?$ & $\begin{array}{l}46 \pm 5 \\
(40-50)\end{array}$ & $\begin{array}{l}42 \pm 6 \\
(35-48)\end{array}$ \\
\hline $\mathrm{a}$ & 25 & 24-29 & 21 & 21.8 & 27.3 & $\begin{array}{c}20.7 \pm 1.7 \\
(19.2-22.6)\end{array}$ & $\begin{array}{c}28.6 \pm 2.4 \\
(25.3-30.6)\end{array}$ \\
\hline $\mathrm{b}$ & $7.5-8.7$ & $7.3-7.4$ & 13 & 6.3 & 9.1 & $\begin{array}{c}9.5 \pm 0.9 \\
(8.7-10.4)\end{array}$ & $\begin{array}{c}9.6 \pm 0.8 \\
(8.6-10.6)\end{array}$ \\
\hline $\mathrm{c}$ & $14.2-17.3$ & $13.6-15.7$ & 21 & 14.2 & 18.5 & $\begin{array}{c}17.5 \pm 1.8 \\
(15.4-18.6)\end{array}$ & $\begin{array}{c}17.1 \pm 1.0 \\
(15.7-18.1)\end{array}$ \\
\hline$c^{\prime}$ & $?$ & $?$ & 1.5 & 1.9 & 1.8 & $\begin{array}{l}1.9 \pm 0.3 \\
(1.7-2.2)\end{array}$ & $\begin{array}{l}2.0 \pm 0.2 \\
(1.8-2.2)\end{array}$ \\
\hline $\mathrm{V}(\%) / \mathrm{T}(\%)$ & $71-73$ & $?$ & 74 & 64 & $?$ & $\begin{array}{c}69.5 \pm 0.6 \\
(69.0-70.2)\end{array}$ & $\begin{array}{c}39.8 \pm 2.0 \\
(37.6-41.4)\end{array}$ \\
\hline Labial region diameter & $?$ & $?$ & 23 & $?$ & $?$ & $\begin{array}{c}14.7 \pm 0.8 \\
(14.0-15.0)\end{array}$ & $\begin{array}{c}14.0 \pm 1.3 \\
(13.0-16.0)\end{array}$ \\
\hline Cephalic setae length & $?$ & 2 & 3 & 2.6 & 2.6 & 3.0 & $\begin{array}{l}2.9 \pm 0.3 \\
(2.5-3.5)\end{array}$ \\
\hline $\begin{array}{l}\text { Subdorsal cephalic setae } \\
\text { from anterior end }\end{array}$ & $?$ & 13 & 23 & $?$ & $?$ & $\begin{array}{c}14.1 \pm 3.4 \\
(12.0-16.5)\end{array}$ & $\begin{array}{c}12.9 \pm 0.7 \\
(12.5-14.0)\end{array}$ \\
\hline $\begin{array}{l}\text { Subventral cephalic setae } \\
\text { from anterior end }\end{array}$ & $?$ & 10 & 18 & $?$ & $?$ & $\begin{array}{c}11.0 \pm 1.2 \\
(10.0-12.0)\end{array}$ & $\begin{array}{c}10.5 \pm 1.3 \\
(10.0-12.5)\end{array}$ \\
\hline $\begin{array}{l}\text { Cephalic setae length / } \\
\text { labial region diam. }\end{array}$ & $?$ & $?$ & $?$ & $?$ & $?$ & 0.2 & $\begin{array}{c}0.2 \\
(0.2-0.3)\end{array}$ \\
\hline $\begin{array}{l}\text { Anterior end of shield } \\
\text { from anterior end }\end{array}$ & $?$ & $?$ & $?$ & $?$ & $?$ & $\begin{array}{c}17.3 \pm 1.4 \\
(16.0-18.5)\end{array}$ & $\begin{array}{c}14.3 \pm 0.9 \\
(13.0-15.0)\end{array}$ \\
\hline Refractive plate length & $?$ & 23 & 27 & 28 & 29 & $\begin{array}{c}22.8 \pm 3.2 \\
(19.0-25.5)\end{array}$ & $\begin{array}{c}23.1 \pm 3.3 \\
(20.0-27.5)\end{array}$ \\
\hline Refractive plate width & $?$ & 13 & 16 & 11.7 & 13.6 & $\begin{array}{c}14.3 \pm 2.4 \\
(12.0-16.5)\end{array}$ & $\begin{array}{c}14.3 \pm 1.0 \\
(13.0-15.0)\end{array}$ \\
\hline $\begin{array}{l}\text { Anterior end of amphid } \\
\text { from anterior end }\end{array}$ & $?$ & $?$ & 19 & $?$ & $?$ & $\begin{array}{c}12.9 \pm 2.2 \\
(10.5-14.5)\end{array}$ & $\begin{array}{c}11.0 \pm 1.1 \\
(10.0-12.5)\end{array}$ \\
\hline $\begin{array}{l}\text { Dorsal amphid branch } \\
\text { length }\end{array}$ & $?$ & 20 & 32 & $?$ & $?$ & $\begin{array}{c}23.7 \pm 2.8 \\
(21.0-26.0)\end{array}$ & $\begin{array}{c}22.9 \pm 2.5 \\
(21.0-26.0)\end{array}$ \\
\hline $\begin{array}{l}\text { Ventral amphid branch } \\
\text { length }\end{array}$ & $?$ & 20 & 28 & $?$ & $?$ & $\begin{array}{l}24.4 \pm 3.3 \\
(21.0-27.0)\end{array}$ & $\begin{array}{c}24.0 \pm 3.2 \\
(21.0-28.0)\end{array}$ \\
\hline Amphid width & $?$ & 4 & 7 & $?$ & $?$ & $\begin{array}{l}5.8 \pm 0.4 \\
(5.5-6.0)\end{array}$ & $\begin{array}{l}5.2 \pm 0.4 \\
(5.5-5.5)\end{array}$ \\
\hline
\end{tabular}


Table 5 (continued).

\begin{tabular}{|c|c|c|c|c|c|c|c|}
\hline \multirow[b]{2}{*}{ Amphid length / width } & \multicolumn{2}{|c|}{ original description } & \multirow{2}{*}{$\begin{array}{c}\begin{array}{c}\text { data for } \\
\text { D. cuspidiboja }\end{array} \\
4.6\end{array}$} & \multicolumn{2}{|c|}{$\begin{array}{c}\text { D. incisa sensu } \\
\text { Vitiello, } 1972\end{array}$} & \multicolumn{2}{|c|}{ recent specimens } \\
\hline & $?$ & $?$ & & $?$ & $?$ & $\begin{array}{l}4.2 \pm 0.7 \\
(3.3-4.8)\end{array}$ & $\begin{array}{l}4.5 \pm 0.7 \\
(3.8-5.4)\end{array}$ \\
\hline $\begin{array}{l}\text { Anterior edge of stoma } \\
\text { from anterior end }\end{array}$ & $?$ & 13 & 16 & 6.5 & 10 & $\begin{array}{c}12.0 \pm 1.7 \\
(10.5-14.0)\end{array}$ & $\begin{array}{l}10.9 \pm 1.7 \\
(9.0-13.0)\end{array}$ \\
\hline Stoma length & $?$ & $?$ & $?$ & $?$ & $?$ & $\begin{array}{c}15.5 \pm 1.5 \\
(14.5-16.5)\end{array}$ & $\begin{array}{c}14.7 \pm 0.9 \\
(14.0-16.0)\end{array}$ \\
\hline Stoma width & $?$ & $?$ & $?$ & $?$ & $?$ & $\begin{array}{l}3.9 \pm 0.4 \\
(3.5-4.0)\end{array}$ & $\begin{array}{l}3.8 \pm 0.4 \\
(3.5-4.0)\end{array}$ \\
\hline $\begin{array}{l}\text { Nerve ring from anterior } \\
\text { end }\end{array}$ & $?$ & $?$ & 122 & $?$ & $?$ & $\begin{array}{c}103 \pm 2 \\
(102-105)\end{array}$ & $\begin{array}{l}98 \pm 10 \\
(91-112)\end{array}$ \\
\hline $\begin{array}{l}\text { Nerve ring from anterior } \\
\text { end }(\% \text { pharynx })\end{array}$ & $?$ & $?$ & 74 & $?$ & $?$ & $\begin{array}{c}66.8 \pm 2.4 \\
(64.5-69.2)\end{array}$ & $\begin{array}{c}64.9 \pm 2.3 \\
(61.5-66.4)\end{array}$ \\
\hline $\begin{array}{l}\text { Secretory-excretory pore } \\
\text { from ant. end }\end{array}$ & $?$ & $?$ & 148 & $?$ & $?$ & $\begin{array}{c}123 \pm 5 \\
(117-127)\end{array}$ & $\begin{array}{c}119 \pm 14 \\
(111-139)\end{array}$ \\
\hline $\begin{array}{l}\text { Secr.-excr. pore from ant. } \\
\text { end }(\% \text { pharynx })\end{array}$ & $?$ & $?$ & 90 & $?$ & $?$ & $\begin{array}{c}79.6 \pm 0.8 \\
(79.0-80.4)\end{array}$ & $\begin{array}{c}78.7 \pm 2.5 \\
(76.4-81.3)\end{array}$ \\
\hline Vagina or spicules length & $?$ & 63 & $?$ & $?$ & 52 & $\begin{array}{l}21.7 \pm 2.0 \\
(19.5-23.0)\end{array}$ & $\begin{array}{c}61.8 \pm 4.0 \\
(57.0-66.0)\end{array}$ \\
\hline $\begin{array}{l}\text { Vagina / } \mathrm{BD} \text { or spicules } \\
\text { / } \mathrm{ABD}\end{array}$ & $?$ & $?$ & $?$ & $?$ & 1.3 & $\begin{array}{l}0.3 \pm 0.1 \\
(0.3-0.4)\end{array}$ & $\begin{array}{l}1.5 \pm 0.1 \\
(1.4-1.6)\end{array}$ \\
\hline $\begin{array}{l}\text { Rectum or gubernaculum } \\
\text { length }\end{array}$ & $?$ & $?$ & $?$ & $?$ & $?$ & $\begin{array}{c}31.5 \pm 4.0 \\
(27.5-35.0)\end{array}$ & $\begin{array}{l}9.7 \pm 1.1 \\
(9.0-11.5)\end{array}$ \\
\hline $\begin{array}{l}\text { Rectum / ABD or } \\
\text { apophysis length }\end{array}$ & $?$ & 10 & $?$ & $?$ & $?$ & $\begin{array}{l}0.7 \pm 0.1 \\
(0.6-0.8)\end{array}$ & $\begin{array}{c}12.8 \pm 1.7 \\
(11.5-15.0)\end{array}$ \\
\hline $\begin{array}{l}\text { Caudal gland openings } \\
\text { from tail terminus }\end{array}$ & $?$ & $?$ & 3.5 & $?$ & $?$ & $\begin{array}{l}5.3 \pm 1.7 \\
(3.5-7.0)\end{array}$ & $\begin{array}{l}6.0 \pm 0.6 \\
(5.5-7.0)\end{array}$ \\
\hline
\end{tabular}

\section{Male}

Reproductive system diorchic, testes opposed; anterior testis outstretched and posterior testis reflexed (cannot be measured). Spicules paired and symmetrical, strongly curved, with ovoid manubrium and fusiform shaft. Gubernaculum plate-like, with pair of strong closely set caudal apophyses variable in shape (Fig. 13C-E). Caudal setae present, several ventrosublateral pairs arranged in rows along entire tail length, one dorsosublateral pair located close to tail terminus (not always visible).

\section{Remarks}

No morphological or morphometric differences can be found between the recent specimens and the original description of this species (Jensen 1991).

Key to species of the genus Neodiplopeltula gen. nov. (see Table 6 for additional diagnostic characters)

1. Refractive periamphideal plates absent N. intermedia (Gerlach, 1954) gen. et comb. nov.

- Refractive periamphideal plates present 2

2. Refractive periamphideal plates connect on dorsal and ventral body sides 3

- Refractive periamphideal plates do not connect 4 
3. Oral opening subdorsal; amphid $32-44 \mu \mathrm{m}$ long $N$. onusta (Wieser, 1956) gen. et comb. nov.

- Oral opening terminal; amphid 16-17 $\mu \mathrm{m}$ long N. obesa (Nguyen Vu Thahn, Nguyen Thahn Hien \& Gagarin, 2012) gen. et comb. nov.

4. Oral opening terminal N. indica (Gerlach 1962) gen. et comb. nov.

- Oral opening subdorsal 5

5. Oral opening at level with or posterior to cephalic setae bases, $6-16 \mu \mathrm{m}$ from anterior end

N. bathmanni (Jensen, 1991) gen. et comb. nov.

- Oral opening anterior to cephalic setae bases, $1-3 \mu \mathrm{m}$ from anterior end 6

6. Refractive periamphideal plates with incised margins; cephalic setae more than $8 \mu \mathrm{m}$ long N. incisa (Southern, 1914) gen. et comb. nov.

- Refractive periamphideal plates oval in shape; cephalic setae less than $8 \mu \mathrm{m}$ long

N. barentsi (Steiner, 1916) gen. et comb. nov.

\section{Amendment to the key to the genera of Diplopeltidae (from Holovachov 2017)}

10. Stoma subcylindrical; caudal glands open via three separate pores Neodiplopeltula gen. nov.

- Stoma small and undifferentiated; caudal glands open via common spinneret .11

11. Amphid located on a well-developed refractive subcuticular plate

Diplopeltis Cobb in Stiles \& Hassal, 1905

- Refractive subcuticular plate absent Pseudaraeolaimus Chitwood, 1951
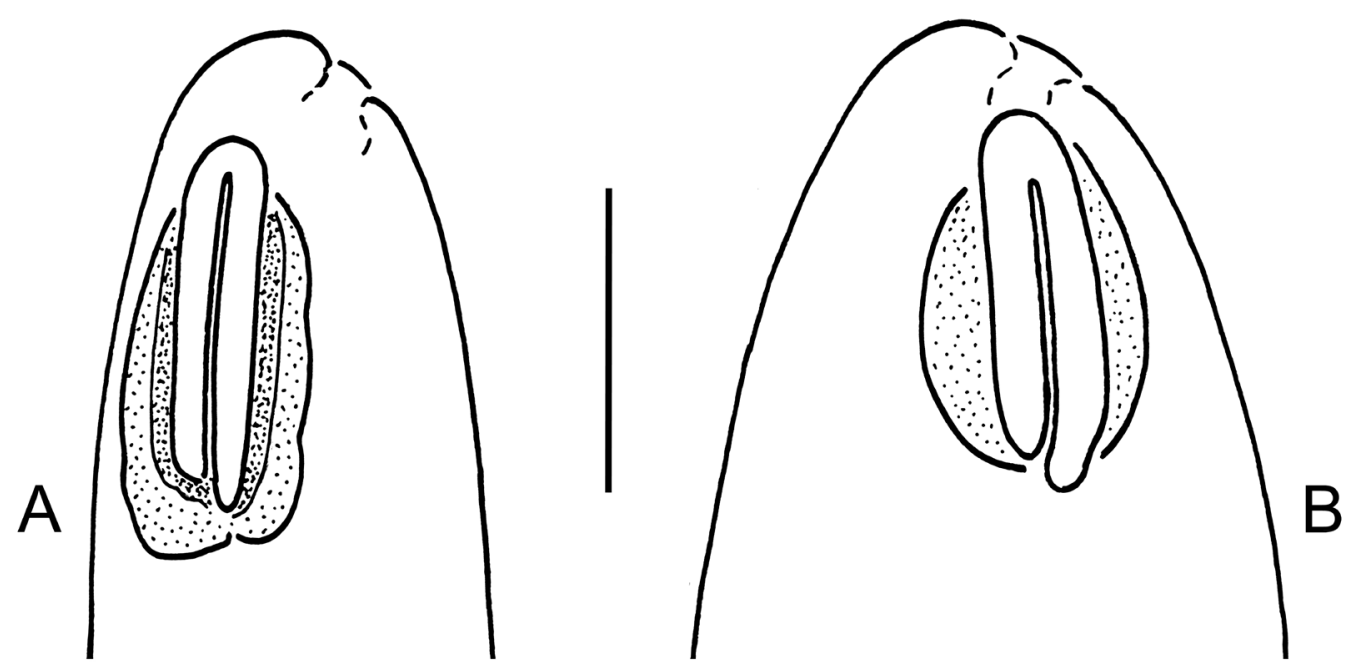

Fig. 14. A. Anterior end of holotype of Diplopeltis ovalis Ditlevsen, 1928. B. Anterior end of lectotype of Diplopeltis asymmetricus Allgén, 1935. Scale bar $=20 \mu \mathrm{m}$. 


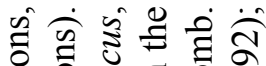

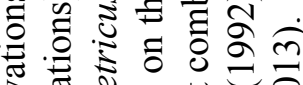

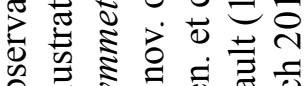

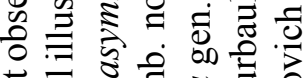

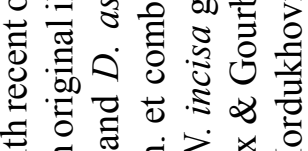

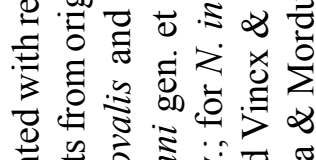

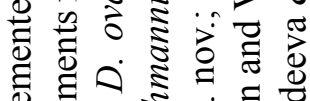

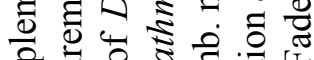

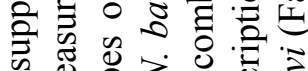

की

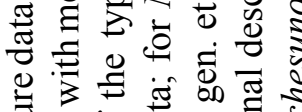

诺菏

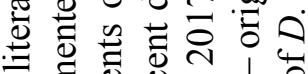

б

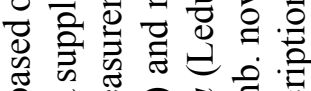

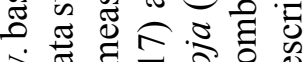

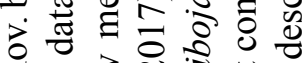

.

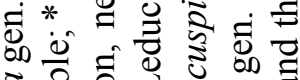

을

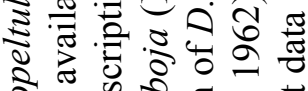

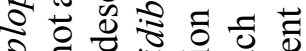

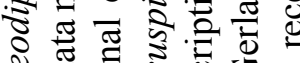

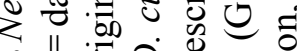

क

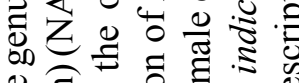

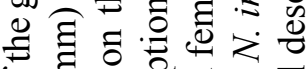

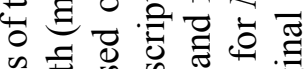

造

送

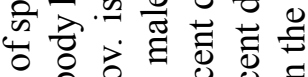

要

过客富

䒕 ¿

웡 屯

के

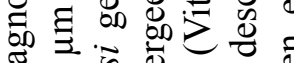

急.

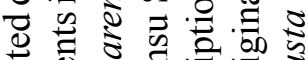

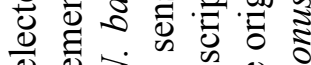

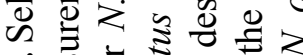

啳

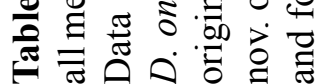

\begin{tabular}{|c|c|c|c|c|c|c|c|}
\hline 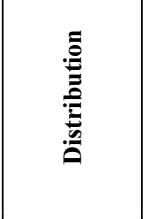 & 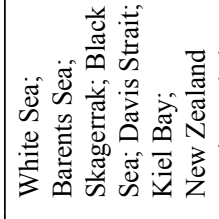 & 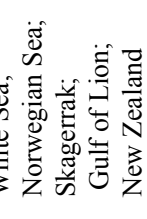 & 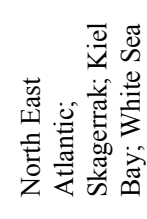 & 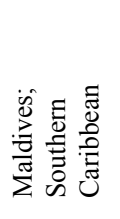 & 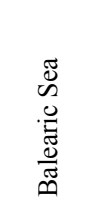 & 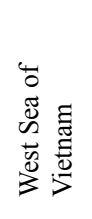 & 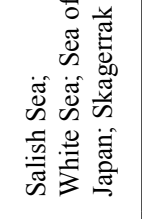 \\
\hline 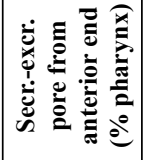 & $\begin{array}{l}8 \\
: \\
\infty \\
i n\end{array}$ & ô & $\begin{array}{l}\vec{b} \\
\dot{b} \\
i\end{array}$ & a. & $\vec{\lambda}$ & r. & $\begin{array}{l}0 \\
\vdots \\
\text { in }\end{array}$ \\
\hline 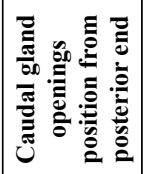 & $I$ & 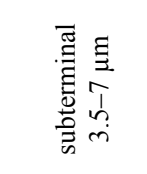 & 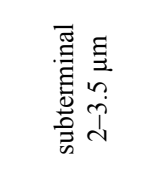 & 产 & $\therefore$ & 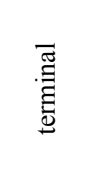 & $\begin{array}{l}\overline{\underline{\underline{w}}} \\
\underline{\underline{\underline{z}}}\end{array}$ \\
\hline$=$ & 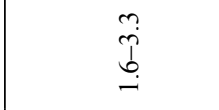 & $\begin{array}{l}\text { İ } \\
\text { in }\end{array}$ & $\begin{array}{l}\text { के } \\
\text { iे }\end{array}$ & 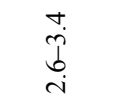 & $\ddot{m}$ & $\begin{array}{l}n \\
\hat{n} \\
n_{n}\end{array}$ & $\begin{array}{l}\text { i } \\
\dot{I} \\
\dot{I}\end{array}$ \\
\hline 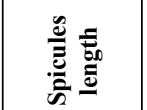 & $\begin{array}{c}0 \\
i \\
y\end{array}$ & $\begin{array}{l}: \\
\vdots \\
i\end{array}$ & 帘 & $\begin{array}{l}0 \\
\vdots \\
\vdots\end{array}$ & $\overleftrightarrow{z}$ & $\overleftarrow{z}$ & $\underset{i}{i}$ \\
\hline$\stackrel{\circ}{\grave{D}}$ & $\begin{array}{l}\text { t } \\
0 \\
0\end{array}$ & $\begin{array}{l}\text { t } \\
\text { f } \\
\text { t }\end{array}$ & $\begin{array}{l}8 \\
\frac{8}{6}\end{array}$ & in & 8 & $\begin{array}{l}\tilde{W} \\
\sigma\end{array}$ & $\begin{array}{l}\infty \\
0 \\
b \\
b\end{array}$ \\
\hline 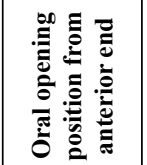 & 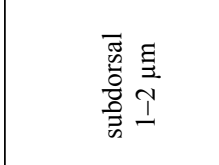 & 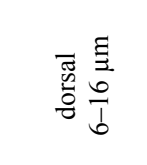 & 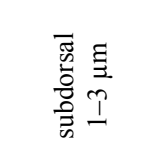 & 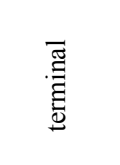 & 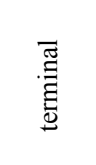 & 首 & 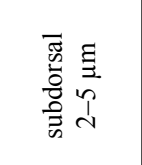 \\
\hline 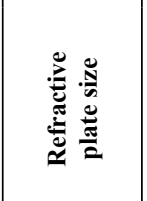 & 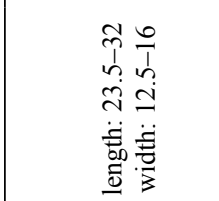 & 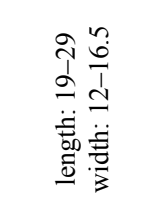 & 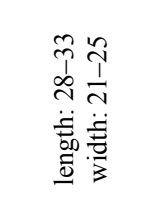 & 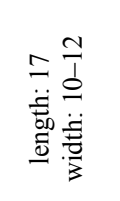 & $\mathbb{z}$ & $\alpha$ & 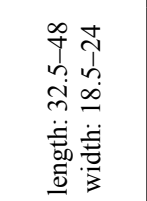 \\
\hline 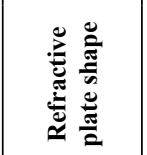 & 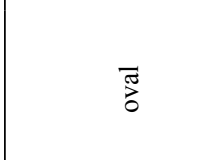 & $\overline{\mathrm{g}}$ & 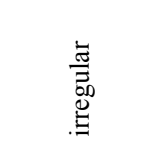 & $\overline{\widetilde{g}}$ & $\begin{array}{l}\text { 言 } \\
\text { 啇 }\end{array}$ & 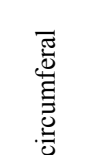 & 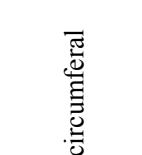 \\
\hline$\frac{8}{\frac{8}{0}}$ & 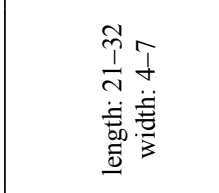 & 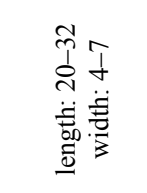 & 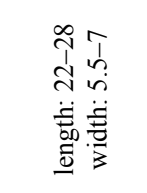 & 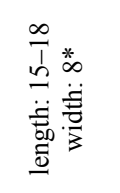 & 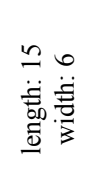 & 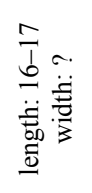 & 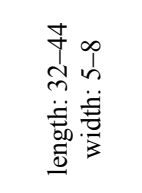 \\
\hline 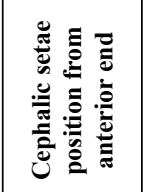 & 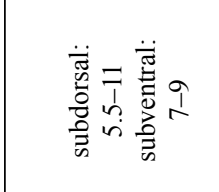 & 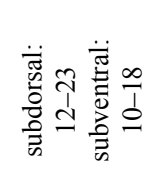 & 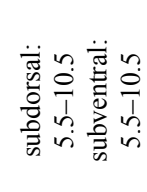 & 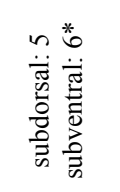 & * & 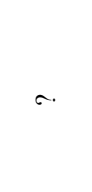 & 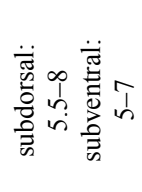 \\
\hline 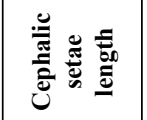 & $\begin{array}{l}n \\
i \\
n \\
n\end{array}$ & $\stackrel{n}{i}$ & $\underset{\infty}{\Delta}$ & $\frac{\Im}{\alpha}$ & 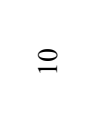 & I & $\begin{array}{l}n \\
\vdots \\
n\end{array}$ \\
\hline 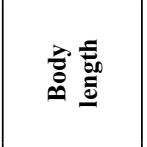 & $\begin{array}{l}5 \\
\dot{j} \\
\dot{t} \\
0 \\
0\end{array}$ & $\begin{array}{l}\infty \\
0 \\
i \\
\infty \\
0 \\
0\end{array}$ & 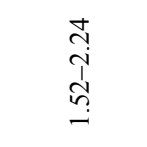 & 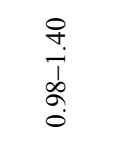 & فํ. & $\begin{array}{l}0 \\
0 \\
0 \\
0 \\
f \\
0\end{array}$ & $\begin{array}{l}\stackrel{\infty}{+} \\
\stackrel{+}{0} \\
0 \\
0\end{array}$ \\
\hline & $\begin{array}{l}\tilde{5} \\
\bar{\Sigma} \\
\bar{\Sigma} \\
z\end{array}$ & 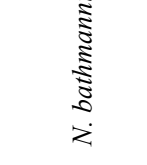 & 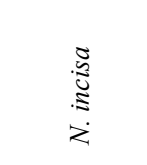 & 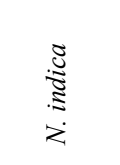 & 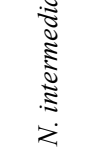 & 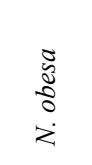 & 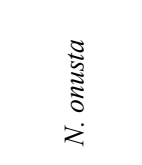 \\
\hline
\end{tabular}




\section{Discussion}

The genus Diplopeltula exhibits two pertinent problems in the systematics of marine nematodes: ignorance of the female morphology in descriptions of new taxa and the absence of type material. The 'Traditional' focus lies on male characters in species descriptions and diagnoses of genera, and the partial or complete ignorance towards the morphology of female reproductive system causes taxonomic and nomenclatorial problems in those cases where higher classification of taxa relies on female-specific characters. The genus Diplopeltula is but one example (see detailed discussions in Holovachov et al. 2009; Leduc 2013; Holovachov 2017; Holovachov \& Boström 2017). Of course, it would not be such an issue if the type material for such 'troublesome' taxa were preserved and available for restudy. Unfortunately, S. Gerlach, being one of the most prolific nematode taxonomists, did not preserve any type specimens and did not make any permanent slides until 1964, thus preventing any future taxonomic revisions and corrections. Regrettably, he was not alone in this practice. As it turned out, W. Wieser did not leave any trace of the enormous and taxonomically important collection of marine nematodes from Chile, consequences of which are yet to be dealt with.

\section{Acknowledgments}

This research was supported by two grants from the Swedish Taxonomy Initiative: "Systematics of Swedish free-living nematodes of the orders Desmodorida and Araeolaimida" and "Systematics of poorly known marine nematodes of the class Chromadorea from Sweden". Sampling in the Skagerrak was conducted using vessels ("Skagerak" and "Oscar von Sydow") and facilities of the Sven Lovén Centre for Marine Sciences in Kristineberg. "Inventering Bratten" was an interdisciplinary inventory project also financially supported by the Swedish Taxonomy Initiative. Authors are grateful to Dr. Laura Pavesi and Prof. Martin V. Sørensen from the Natural History Museum of Denmark in Copenhagen and to Dr. Baptiste Vivier and Dr. Cédric Hubas from the National Museum of Natural History in Paris for making type specimens of respectively Diplopeltis ovalis Ditlevsen, 1928 and Diplopeltula ostrita Boucher \& Helléouët, 1977 available for study.

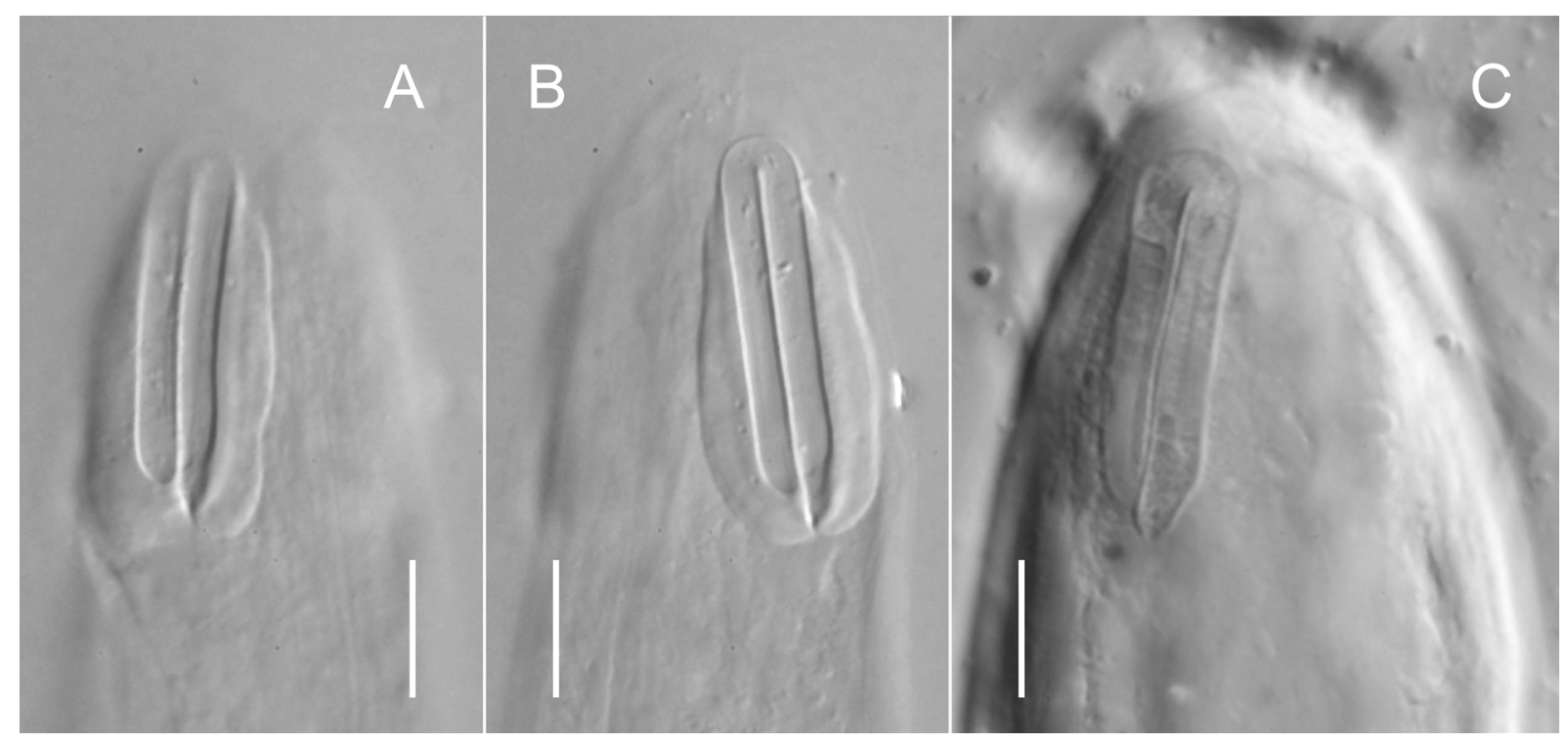

Fig. 15. A-B. Anterior end of the holotype of Diplopeltis ovalis Ditlevsen, 1928. C. Anterior end of the lectotype of Diplopeltis asymmetricus Allgén, 1935. Scale bar $=10 \mu \mathrm{m}$. 


\section{References}

Allgén C. 1935. Die freilebenden Nematoden des Öresunds. Capita Zoologica 6: 1-192.

De Grisse A.T. 1969. Redescription ou modifications de quelques techniques utilisées dans l'étude des nématodes phytoparasitaires. Mededelingen van de Rijksfakulteit voor Landbouwwetenschappen Gent 34: 351-369.

Ditlevsen H. 1928. Free-living marine nematodes from Greenland waters. Meddelelser om Grønland 23 (Suppl.): 199-250.

Fadeeva N. \& Mordukhovich V. 2013. Some new and poorly known nematode species from the Sea of Japan. Deep-Sea Research II 86-87: 119-123. https://doi.org/10.1016/j.dsr2.2012.07.044

Gerlach S.A. 1950. Die Diplopeltiden, eine Gruppe freilebender Nematoden. Kieler Meeresforschungen 7: $138-156$.

Holovachov O. 2017. Belgopeltula belgica (Vincx \& Gourbault, 1992) gen. et comb. nov. and Mudwigglus micramphidium sp. nov. from the west coast of Sweden, and reappraisal of the genus Pseudaraeolaimus Chitwood, 1951 (Nematoda: Araeolaimida: Diplopeltidae). European Journal of Taxonomy 383: 1-21. https://doi.org/10.5852/ejt.2017.383

Holovachov O. \& Boström S. 2017. Three new and five known species of Diplopeltoides Gerlach, 1962 (Nematoda, Diplopeltoididae) from Sweden, and a revision of the genus. European Journal of Taxonomy 369: 1-35. https://doi.org/10.5852/ejt.2017.369

Holovachov O., Tandingan De Ley I., Mundo-Ocampo M., Gingold R \&, De Ley P. 2009. Nematodes from the Gulf of California. Part 3. Three new species of the genus Diplopeltoides Gerlach, 1962 (Nematoda: Diplopeltoididae) with overviews of the genera Diplopeltis Gerlach, 1962 and Diplopeltula Gerlach, 1950. Russian Journal of Nematology 17: 43-57.

Jensen P. 1991. Nine new and less known nematode species from the deep-sea benthos of the Norwegian Sea. Hydrobiologia 222: 57-76. https://doi.org/10.1007/BF00017500

Leduc D. 2013. Mudwigglus gen. n. (Nematoda: Diplopeltidae) from the continental slope of New Zealand, with description of three new species and notes on their distribution. Zootaxa 3682: 351-370. https://doi.org/10.11646/zootaxa.3682.2.8

Leduc D. 2017. Four new nematode species (Araeolaimida: Comesomatidae, Diplopeltidae) from the New Zealand continental slope. Zootaxa 4237: 244-264. https://doi.org/10.11646/zootaxa.4237.2.2

Maggenti A.R. 2005. Online Dictionary of Invertebrate Zoology. Available from digitalcommons.unl.edu [accesed 13 Aug. 2018].

Seinhorst J.W. 1959. A rapid method for the transfer of nematodes from fixative to anhydrous glycerin. Nematologica 4: 67-69. https://doi.org/10.1163/187529259X00381

Sergeeva N.G. 1977. Free-living nematoda, new for the Black Sea. Vestnik Zoologii 1: 36-44.

Southern R. 1914. Nemathelmaia, Kinorhyncha, and Chaetognatha (Clare Island Survey, Part 54). Proceedings of the the Royal Irish Academy 31: 1-80.

Steiner G. 1916. Freilebende Nematoden aus der Barentssee. Zoologische Jahrbücher. Abteilung für Systematik, Geographie und Biologie der Tiere 39: 511-664.

Vincx M. \& Gourbault N. 1992. Six new species of the genus Diplopeltula (Nematoda: Diplopeltidae) with remarks on the heterogeneity of the taxon. Hydrobiologia 230: 165-178.

https://doi.org/10.1007/BF00036563 
Vitiello P. 1972. Sur quelques espèces de Diplopeltula (Nematoda, Araeolaimida). Vie et Milieu 21: $535-544$.

Voronov D.A. 1982. [Free-living nematodes of the genus Diplopeltula Gerlach, 1950 (Araeolaimida, Axonolaimidae) from the White Sea.] Sovremennye problemy biogeografii: 189-197. [in Russian.]

Wieser W. 1956. Free-living Nematodes and Other Small Invertebrates of Puget Sound Beaches. University of Washington Press.

Manuscript received: 13 April 2018

Manuscript accepted: 28 June 2018

Published on: 29 August 2018

Topic editor: Rudy Jocqué

Desk editor: Pepe Fernández

Printed versions of all papers are also deposited in the libraries of the institutes that are members of the EJT consortium: Muséum national d'Histoire naturelle, Paris, France; Botanic Garden Meise, Belgium; Royal Museum for Central Africa, Tervuren, Belgium; Natural History Museum, London, United Kingdom; Royal Belgian Institute of Natural Sciences, Brussels, Belgium; Natural History Museum of Denmark, Copenhagen, Denmark; Naturalis Biodiversity Center, Leiden, the Netherlands; Museo Nacional de Ciencias Naturales-CSIC, Madrid, Spain; Real Jardín Botánico de Madrid CSIC, Spain; Zoological Research Museum Alexander Koenig, Bonn, Germany. 